\title{
Design, Synthesis, and Antifungal Activity of New $\alpha$-Aminophosphonates
}

\author{
Zahra Rezaei, ${ }^{1}$ Soghra Khabnadideh, ${ }^{1}$ Kamiar Zomorodian, ${ }^{2}$ Keyvan Pakshir, ${ }^{2}$ \\ Setareh Nadali, ${ }^{1}$ Nadia Mohtashami, ${ }^{1}$ and Ehsan Faghih Mirzaei ${ }^{1,3}$ \\ ${ }^{1}$ Department of Medicinal Chemistry and Pharmaceutical Sciences Research Center, Faculty of Pharmacy,
Shiraz University of Medical Sciences, Shiraz 71345, Iran
${ }^{2}$ Department of Parasitology, Faculty of Medicine, Shiraz University of Medical Sciences, Shiraz, Iran
${ }^{3}$ Department of Medicinal Chemistry, Faculty of Pharmacy, Kerman University of Medical Sciences, Kerman, Iran
}

Correspondence should be addressed to Zahra Rezaei, rezaeiza@sums.ac.ir

Received 18 May 2011; Revised 29 June 2011; Accepted 13 July 2011

Academic Editor: Stewart Schneller

Copyright ( $) 2011$ Zahra Rezaei et al. This is an open access article distributed under the Creative Commons Attribution License, which permits unrestricted use, distribution, and reproduction in any medium, provided the original work is properly cited.

$\alpha$-Aminophosphonates are bioisosteres of amino acids and have several pharmacological activities. These compounds have been synthesized by various routes from reaction between amine, aldehyde, and phosphite compounds. In order to synthesize $\alpha$ aminophosphonates, catalytic effect of $\mathrm{CuCl}_{2}$ was compared with $\mathrm{FeCl}_{3}$. Also all designed structures as well as griseofulvin were docked into the active site of microtubule (1JFF), using Autodock program. The results showed that the reactions were carried out in the presence of $\mathrm{CuCl}_{2}$ in lower yields, and also the time of reaction was longer in comparison with $\mathrm{FeCl}_{3}$. The chemical structures of the new compounds were confirmed by spectral analyses. The compounds were investigated for antifungal activity against several fungi in comparison with griseofulvin. An indole-derived bis( $\alpha$-aminophosphonates) with the best negative $\Delta \mathrm{G}$ in docking study showed maximum antifungal activity against Microsporum canis, and other investigated compounds did not have a good antifungal activity.

\section{Introduction}

The $\alpha$-aminophosphonates are amino acid analogues, which have found a wide range of applications in the areas of industrial, agricultural, and medicinal chemistry owing to their biological and physical properties as well as their utility as synthetic intermediates [1-5]. As a kind of natural amino acid analogues, $\alpha$-aminophosphonates constitute an important class of compounds with diverse biological activities. The activity of $\alpha$-aminophosphonates as pharmacogenic agents [6] is reported in the literature. Also it has been reported that some alkyl-substituted phosphonate compounds have antifungal activity $[7,8]$, antibacterial activity $[9,10]$, antitumor effects [11-13], and antiviral activity [14].

Three-component synthesis starting from aldehydes, amines and diethyl phosphite or triethyl phosphite have been reported by using Lewis and Bronsted acid catalysts such as $\mathrm{LiClO} 4$ [15], $\mathrm{InCl}_{3}$ [16], $\mathrm{AlCl}_{3}$ [17], lanthanide triflates/magnesium sulfate [18], $\mathrm{SbCl}_{3} / \mathrm{Al}_{2} \mathrm{O}_{3}$ [19], $\mathrm{TaCl}_{5}-\mathrm{SiO}_{2}$
[20], $\mathrm{CF}_{3} \mathrm{CO}_{2} \mathrm{H}$ [21], scandium (tris-dodecyl sulfate) [22], $\mathrm{BF}_{3} \cdot \mathrm{Et}_{2} \mathrm{O}$ [23], $\mathrm{M}(\mathrm{OTf})_{n}$ [24], and $\mathrm{M}\left(\mathrm{ClO}_{4}\right)_{n}$ [25], though, many of these methods suffer from some drawbacks such as long reaction times, low yields of the products, requiring stoichiometric amounts of catalysts, costly and moisture sensitive catalysts, and use of highly toxic or toxic catalysts. More recently, $\mathrm{ZrOCl}_{2} \cdot 8 \mathrm{H}_{2} \mathrm{O}$ [26] or $\mathrm{ZrO}\left(\mathrm{ClO}_{4}\right)_{2} \cdot 6 \mathrm{H}_{2} \mathrm{O}$ [27] and $\mathrm{TiO}_{2}$ [28] are reported to be effective catalysts for the formation of $\alpha$-aminophosphonates using a threecomponent system composing of aldehydes/ketones, amines, and diethylphosphite under neat conditions. Recently, we have reported one-pot three-component synthesis starting from aldehydes, amines and diethylphosphite using $\mathrm{FeCl}_{3}$ as a catalyst to formation of $\alpha$-aminophosphonates [29]. As $\mathrm{FeCl}_{3}$ suffers from being hygroscopic and is also a corrosive material, in this study the catalyst effect of $\mathrm{CuCl}_{2}$ was compared with $\mathrm{FeCl}_{3}$ for $\alpha$-aminophosphonates preparation.

As it has been reported that $\alpha$-aminophosphonates have antifungal and cytotoxic activity [7, 8, 29], in this 


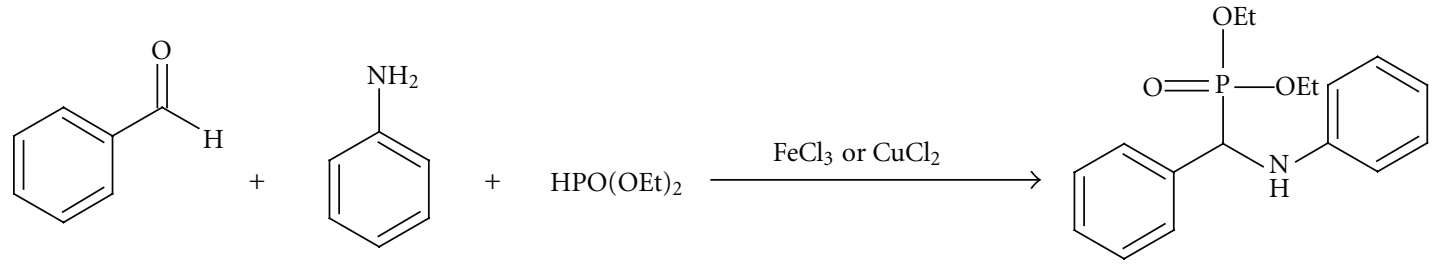

Scheme 1: Three-component reaction of aromatic aldehydes with amine and diethylphosphite.

TABLE 1: Comparison of the effect of catalysts in preparation of $\alpha$-aminophosphonate by the reaction of an aldehyde, aniline and diethylphosphite.

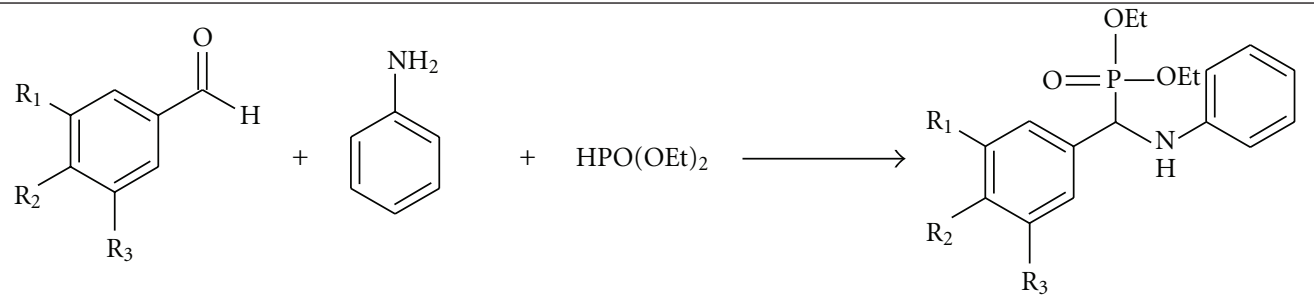

\begin{tabular}{lcccc}
\hline Entry & Catalyst $(0.1 \mathrm{mmol})$ & Solvent & Time $(\mathrm{min})$ & Yield $(\%)$ \\
\hline $\mathbf{1}$ & $\mathrm{FeCl}_{3}$ & THF & $30-120$ & $73-84$ \\
$\mathbf{2}$ & $\mathrm{CuCl}_{2}$ & THF & 24 & $<5 \%$ \\
\hline
\end{tabular}

Compound 1: $\mathrm{R}_{1}=\mathrm{OMe}, \mathrm{R}_{2}=\mathrm{OH}, \mathrm{R}_{3}=\mathrm{H}$; Compound 8: $\mathrm{R}_{1}=$ Ome, $\mathrm{R}_{2}=\mathrm{Ome}, \mathrm{R}_{3}=\mathrm{H}$; Compound 11: $\mathrm{R}_{1}=\mathrm{OMe}, \mathrm{R}_{2}=\mathrm{OMe}, \mathrm{R}_{3}=\mathrm{OMe}$ Compound 12: $\mathrm{R}_{1}=\mathrm{H}, \mathrm{R}_{2}=\mathrm{OMe}, \mathrm{R}_{3}=\mathrm{H}$; Compound 14: $\mathrm{R}_{1}=\mathrm{H}, \mathrm{R}_{2}=\mathrm{H}, \mathrm{R}_{3}=\mathrm{H}$; Compound 20: $\mathrm{R}_{1}=\mathrm{H}, \mathrm{R}_{2}=\mathrm{NO}_{2}, \mathrm{R}_{3}=\mathrm{H}$.

study a series of $\alpha$-aminophosphonates was designed having aromatic aldehydes and amines with $\mathrm{Cl}$ and methoxy moiety similar to griseofulvin structure. Griseofulvin inhibits the growth of fungal cells by inducing abnormal mitosis. It has been reported that griseofulvin blocked the cells at G2/M phase of cell cycle and caused a significant depolymerisation of the spindle microtubules [30]. Because the griseofulvin binding site partially overlaps with the paclitaxel site in tubulin [30], therefore, microtubul complexed with paclitaxel (1JFF) was obtained from Protein Data Bank for docking studies. Autodock program used and all designed structures as well as griseofulvin were docked into the active site of 1JFF. In addition, we synthesized and investigated antifungal activity of some new $\alpha$-aminophosphonates in comparison with griseofulvin.

\section{Results and Discussion}

2.1. Chemistry. In order to synthesize $\alpha$-aminophosphonates, the three components, aldehyde (benzaldehyde, $5.0 \mathrm{mmol}$ ), aromatic amine (aniline, $5.0 \mathrm{mmol}$ ), and diethyl phosphate $(5.5 \mathrm{mmol})$, were reacted in the presence of catalytic amount $(0.1 \mathrm{mmol})$ of $\mathrm{FeCl}_{3}$ or $\mathrm{CuCl}_{2}$ (Scheme 1$)$. The reaction completely proceeded after $90 \mathrm{~min}$ with $73 \%$ yield in the presence of $\mathrm{FeCl}_{3}$, but the reaction did not completely proceed even after $24 \mathrm{~h}$ using $\mathrm{CuCl}_{2}$. The reactions were repeated with several aldehydes, amines, and diethyl phosphates with similar molar ratios as above in the presence of catalytic amount of $\mathrm{FeCl}_{3}$ or $\mathrm{CuCl}_{2}$. The reactions proceeded between $30-120 \mathrm{~min}$ in excellent isolated yields $(73-84 \%)$ in the presence of $\mathrm{FeCl}_{3}$, but $\mathrm{CuCl}_{2}$ was not an effective catalyst like $\mathrm{FeCl}_{3}$. The results of this study are summarized in Table 1.

In this study 21 compounds were synthesized. The synthesis of compounds $\mathbf{1}, \mathbf{8}, \mathbf{1 1}, \mathbf{1 2}, \mathbf{1 4}$, and 20 was carried out in the presence of catalytic amount of $\mathrm{FeCl}_{3}$ or $\mathrm{CuCl}_{2}$ (Table 1). The reactions proceed between 30$120 \mathrm{~min}$ in excellent isolated yields (73-84\%) using $\mathrm{FeCl}_{3}$, but the reaction takes $24 \mathrm{~h}$ using $\mathrm{CuCl}_{2}$. However, it has been reported that metal chloride or metal halide are efficient catalyst for preparation of aminophosphonate by threecomponent reaction [31] but it seems that $\mathrm{CuCl}_{2}$ is not very efficient catalyst for formation of $\alpha$-aminophosphonates in this condition. As our aim was comparison of the catalytic effect of $\mathrm{CuCl}_{2}$ with $\mathrm{FeCl}_{3}$ under same conditions, hence, other conditions were ignored. All compounds were synthesized by one-pot three-component synthesis using $\mathrm{FeCl} 3$ as a catalyst. The reactions completely proceeded after $30-180 \mathrm{~min}$ in excellent isolated yields $(68-90 \%)$ in the presence of $\mathrm{FeCl}_{3}$ (Table 2).

The recommended mechanism for preparation of $\alpha$ aminophosphonates using $\mathrm{FeCl}_{3}$ as a catalyst is shown in Figure 1. As shown in Figure 1, the reaction starts with activation of diethylphosphite a tautomer form in which the $\mathrm{P}(\mathrm{V})$ turns to $\mathrm{P}$ (III) with a free par of electron. Then the nitrogen of Schiff base that is formed in the first step of $\alpha$ aminophosphonates formation donates a pair of electron to make a coordinante bond with $\mathrm{FeCl}_{3}$. This makes nitrogen positively charged which induces partial positive charge on $\mathrm{sp}^{2}$ carbon. The free pair of electrons of phosphorus attacks to the partially positively charged carbon and a cyclic current 
TABLE 2: $\mathrm{FeCl}_{3}$-THF solution catalyzed synthesis of bis( $\alpha$-aminophosphonates) by using a three-component system.

Entry Aldehyde Amine $\quad \alpha$-Aminophosphonate $\quad \begin{gathered}\text { Time } \\ \text { (minutes) }\end{gathered}$

1<smiles>COc1cc(C=O)ccc1O</smiles><smiles>Nc1ccccc1</smiles>

2<smiles>COc1cc(C=O)cc(OC)c1O</smiles><smiles>Nc1ccc([N+](=O)[O-])cc1</smiles>

3<smiles>COc1cc(C=O)ccc1O</smiles><smiles>Nc1ccc([N+](=O)[O-])cc1</smiles>

4<smiles>COc1cc(C=O)cc(OC)c1OC</smiles><smiles>COc1ccc(N)cc1OC</smiles>

5<smiles>COc1ccc(C=O)cc1OC</smiles><smiles>COc1cc(C=O)cc(OC)c1OC</smiles><smiles>COc1ccc(C=O)cc1</smiles><smiles>COc1ccc(C=O)cc1OC</smiles><smiles>COc1cc(C=O)ccc1O</smiles>

10<smiles>COc1cc(C=O)ccc1O</smiles><smiles>Nc1ccc([N+](=O)[O-])cc1</smiles><smiles>Cc1ccc(Cl)cc1N</smiles><smiles>Cc1ccc(Cl)cc1N</smiles><smiles>Nc1ccc(Cl)cc1[N+](=O)[O-]</smiles><smiles>COc1ccc(N)cc1OC</smiles><smiles>Nc1ccccc1</smiles><smiles>COc1cc(C(Nc2ccccc2)P(=O)(O)O)ccc1O</smiles>

120<smiles>COc1cc(C(Nc2ccc([N+](=O)[O-])cc2)P(=O)([O-])O)cc(OC)c1OC</smiles><smiles>COc1cc(C(Nc2ccc([N+](=O)[O-])cc2)P(=O)([O-])O)ccc1O</smiles><smiles>COc1ccc(NC(c2cc(OC)c(OC)c(OC)c2)P(=O)(O)O)cc1OC</smiles><smiles>COc1ccc(C(Nc2ccc([N+](=O)[O-])cc2)P(=O)([O-])O)cc1OC</smiles><smiles>COc1cc(C(Nc2cc(Cl)ccc2C)P(=O)(O)O)cc(OC)c1OC</smiles>

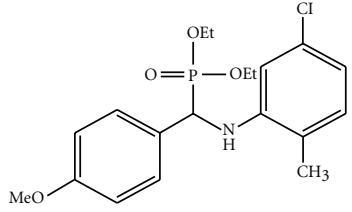<smiles>COc1ccc(C(Nc2ccccc2)P(=O)(O)O)cc1OC</smiles><smiles>COc1cc(C(Nc2ccc(Cl)cc2[N+](=O)[O-])P(=O)(O)O)ccc1O</smiles><smiles>COc1ccc(NC(c2ccc(OC)c(OC)c2)P(=O)(O)O)cc1OC</smiles> 
TABle 2: Continued.

\begin{tabular}{|c|c|c|c|c|c|}
\hline Entry & Aldehyde & Amine & $\alpha$-Aminophosphonate & $\begin{array}{c}\text { Time } \\
\text { (minutes) }\end{array}$ & $\begin{array}{c}\text { Yield } \\
(\%)\end{array}$ \\
\hline 11 & & & & 30 & 81 \\
\hline 12 & & & & 60 & 76 \\
\hline 13 & & & & 90 & 75 \\
\hline 14 & & & & 90 & 73 \\
\hline 15 & & & & 45 & 75 \\
\hline 16 & & & & 90 & 71 \\
\hline 17 & & & & 90 & 70 \\
\hline 18 & & & & 120 & 70 \\
\hline 19 & & & & 120 & 76 \\
\hline 20 & & & & 120 & 73 \\
\hline
\end{tabular}


Table 2: Continued.

Entry Aldehyde Amine

TABLE 3: Docking results of synthesized compounds into the active site of microtubule (1JFF).

\begin{tabular}{lccc}
\hline Entry & $\Delta \mathrm{G}(\mathrm{kcal} / \mathrm{mol})$ & Entry & $\Delta \mathrm{G}(\mathrm{kcal} / \mathrm{mol})$ \\
\hline $\mathbf{1}$ & -6.09 & $\mathbf{1 2}$ & -5.55 \\
$\mathbf{2}$ & -6.18 & 13 & -5.88 \\
$\mathbf{3}$ & -6.16 & 14 & -5.79 \\
$\mathbf{4}$ & -6.23 & 15 & -5.49 \\
$\mathbf{5}$ & -6.36 & 16 & -6.22 \\
$\mathbf{6}$ & -6.27 & 17 & -6.34 \\
$\mathbf{7}$ & -6.71 & $\mathbf{1 8}$ & -6.10 \\
$\mathbf{8}$ & -6.06 & 19 & -6.61 \\
$\mathbf{9}$ & -5.78 & 20 & -6.74 \\
$\mathbf{1 0}$ & -6.27 & 21 & -7.4 \\
$\mathbf{1 1}$ & -6.23 & Griseofulvin & -6.76 \\
\hline
\end{tabular}

of electron displacement protonates nitrogen and detaches the $\mathrm{FeCl}_{3}$ to enter the new cycle. It seems that $\mathrm{CuCl}_{2}$ is not efficient as $\mathrm{FeCl}_{3}$ for attending to this mechanism for formation of $\alpha$-aminophosphonates.

2.2. Modeling. All the compounds (Table 2) as well as griseofulvin were docked into the active site of microtubule, which was obtained from Protein Data Bank (1JFF) using Autodock 4.2. All synthesized compounds were characterized by a docking mode in the active site of the microtubule. Compound 21 showed cytotoxic activity in our previous study [29]. However, this compound has indole moiety like vinca alkaloids but binds to the paclitaxel site in 1JFF like griseofulvin (Figure 2). Therefore, antifungal activity of this compound was investigated in comparison with griseofulvin. According to obtained $\Delta \mathrm{G}$, compound 21 had the maximum negative $\Delta \mathrm{G}$ and compound $\mathbf{1 5}$ had the lowest negative $\Delta \mathrm{G}$ (Table 3); other compounds had $\Delta \mathrm{G}$ close to griseofulvin. Although compound $\mathbf{2 1}$ with maximum negative $\Delta \mathrm{G}$ had the best MIC but there was no correlation between antifungal activity and $\Delta \mathrm{G}$ for other compounds.

2.3. Biological Assay. The synthesized compounds $\mathbf{1}-\mathbf{2 1}$ were evaluated for antifungal activity against Candida albicans, Candida tropicalis, Aspergillus flavus, Microsporum canis, Microsporum gypseum, Trichophyton mentagrophyte, and Epidermophyton floccosum. Agar dilution assay and microdilution method were used to establish the Minimum Inhibitory
Concentration (MIC). The results are presented in Table 4. As shown in Table 4 compounds 1, 7, and 9 showed very low antifungal activity against Trichophyton mentagrophytes. Compound $\mathbf{1}$ also showed very low antifungal activity against Microsporum gypseum. Compound $\mathbf{2 1}$ was the most active compound against Microsporum canis. This compound was previously evaluated in vitro for cytotoxicity effect and showed moderate cytotoxicity activity [29]; here this compound was evaluated for antifungal activity the MIC value found $5 \mu \mathrm{g} / \mathrm{mL}$, and the MIC for compound 21 was better than MIC for griseofulvin. Compound 21 is a bisphosphonate, and it has an indole ring system, perhaps this moiety causes its antifungal activity. Also this compound had the better $\Delta \mathrm{G}$ in docking study. Nevertheless, it has been reported that aminophosphonates have antifungal activity against phytopathogenic fungi $[8,14]$; our synthesized compounds did not show antifungal activity against tested human pathogenic fungi. Song and coworkers reported that antifungal activity of aminophosphonates is related to stereochemistry of them [8]; therefore, may be the antifungal activity of our compound is related to stereochemistry of them. Therefore, we suggest that antifungal evaluation should be done for each enantiomer separately.

\section{Experimental}

All solvents and reagents were purchased from Sigma or Merck Chemical Companies. The products were purified by column chromatography techniques. NMR spectra were recorded on a Brucker Avance DPX 500 MHZ instrument. Mass spectra were recorded on a Hewlett-Packard GC-MS.

3.1. General Procedures for the Synthesis of Compounds. To a mixture of aldehyde $(2 \mathrm{mmol})$, amine $(1 \mathrm{mmol})$, and diethylphosphite $(2.2 \mathrm{mmol})$ was added $\mathrm{FeCl} 3$ in THF $(0.1 \mathrm{mmol})$ and stirred at $60^{\circ} \mathrm{C}$ for the appropriate reaction time. After completion of the reaction, EtOAc $(10 \mathrm{~mL})$ was added to the mixture. The mixture was washed with $\mathrm{H}_{2} \mathrm{O}(10 \mathrm{~mL})$. The organic phase was separated and dried over anhydrous $\mathrm{Na}_{2} \mathrm{SO}_{4}$. The solvent was evaporated in vacuo, and the resulting crude material was purified by chromatography on a short column of silica gel (EtOAc/petroleum ether, 1/3) and then recrystallized from petroleum benzine/dichloromethane (4/1) to afford the pure $\alpha$-aminophosphonates. 


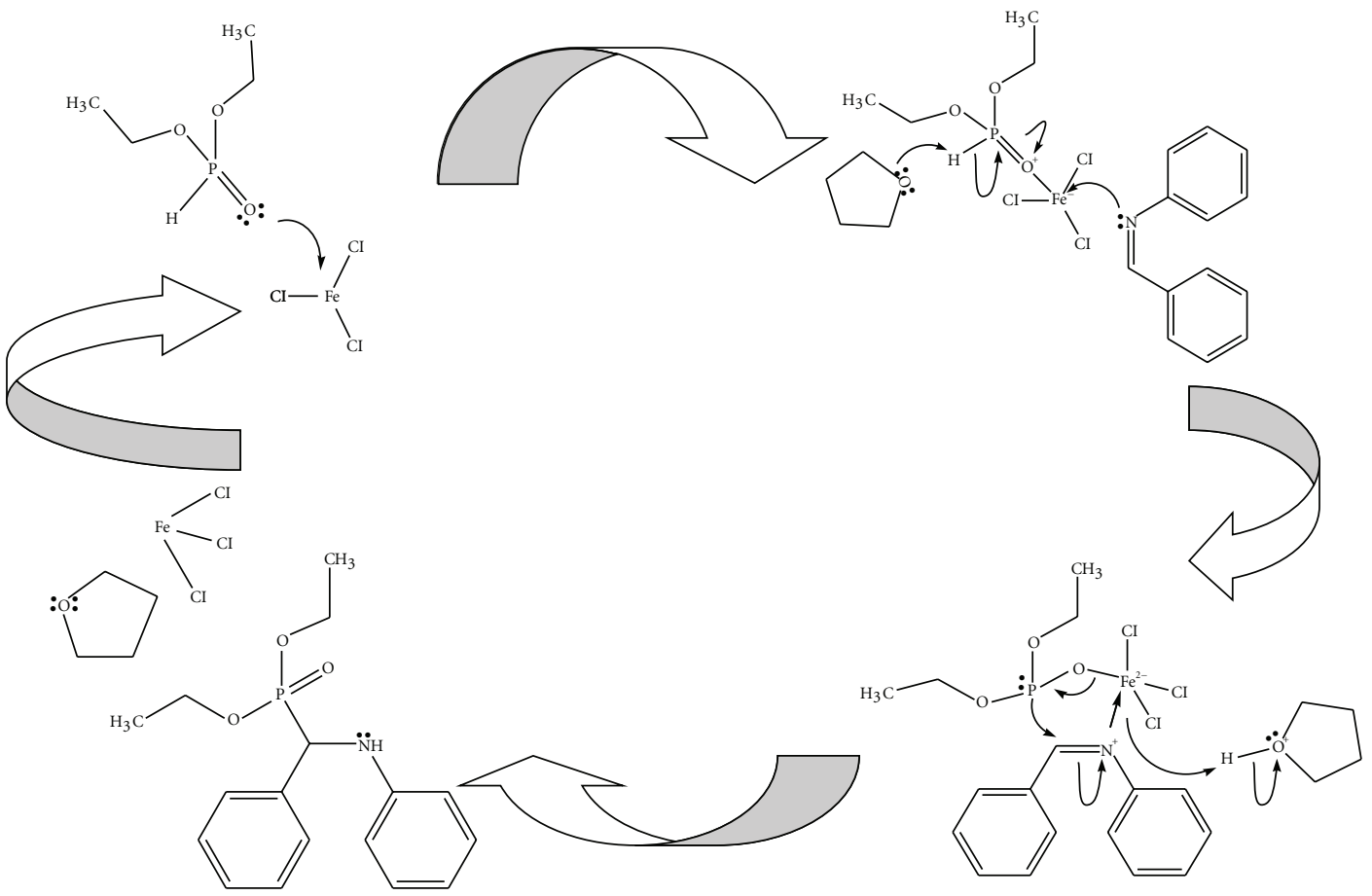

Figure 1: Proposed mechanism for catalytic effect of $\mathrm{FeCl}_{3}$.

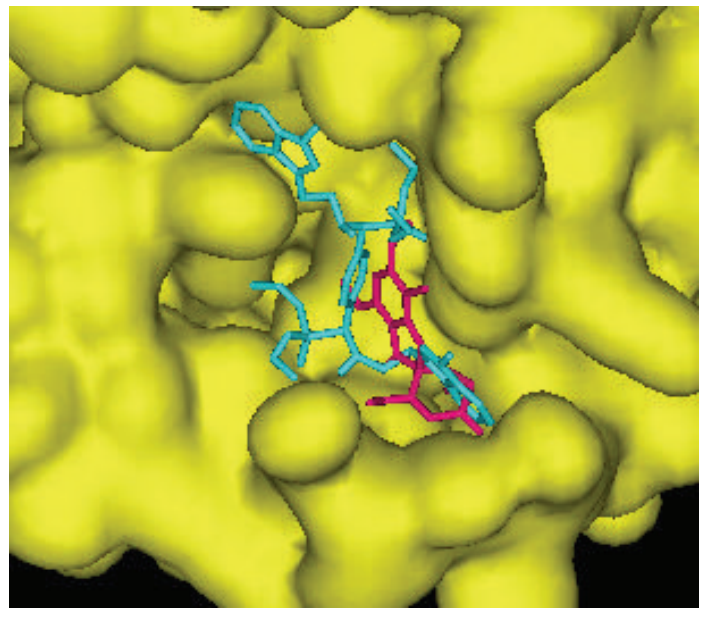

FIGURE 2: Accommodation of Griseofulvin (red) and compound 21 (blue) in the active site of 1JFF.

Diethyl [Anilino(4-hydroxy-3-methoxyphenyl)methyl]phosphonate (1). This compound was synthesized after $120 \mathrm{~min}$ $\left(84 \% . \mathrm{Mp}=95.4^{\circ} \mathrm{C}\right) \cdot{ }^{1} \mathrm{H} \mathrm{NMR}\left(500 \mathrm{MHz}, \mathrm{CDCl}_{3}\right): 7.15$ $(\mathrm{t}, 2 \mathrm{H}, J=6.6 \mathrm{~Hz}, \operatorname{ArH}), 7.04(\mathrm{~s}, 1 \mathrm{H}, \operatorname{ArH}), 6.98(\mathrm{~d}, 1 \mathrm{H}$, $J=6.6 \mathrm{~Hz}, \mathrm{ArH}), 6.90(\mathrm{~d}, 1 \mathrm{H}, J=8.3 \mathrm{~Hz}, \mathrm{ArH}), 6.74(\mathrm{t}, 1 \mathrm{H}$, $J=6.6 \mathrm{~Hz}, \mathrm{ArH}), 6.64(\mathrm{~d}, 2 \mathrm{H}, J=6.6 \mathrm{~Hz}, \mathrm{ArH}), 4.72(\mathrm{~d}$, $\left.1 \mathrm{H}, J_{\mathrm{CHPO}}=21.6 \mathrm{~Hz}, \mathrm{CHP}\right), 4.12-4.18\left(\mathrm{~m}, 2 \mathrm{H}, \mathrm{OCH}_{2} \mathrm{CH}_{3}\right)$, 3.97-4.02 (m, s, $\left.1 \mathrm{H}, \mathrm{OCH}_{2} \mathrm{CH}_{3}\right), 3.87$ (s, 3H, $\left.\mathrm{OCH}_{3}\right), 3.72-$ $3.77\left(\mathrm{~m}, 1 \mathrm{H}, \mathrm{OCH}_{2} \mathrm{CH}_{3}\right), 1.32\left(\mathrm{t}, 3 \mathrm{H}, J=8.3 \mathrm{~Hz}, \mathrm{CH}_{3}\right)$, $1.18\left(\mathrm{t}, 3 \mathrm{H}, J=8 . \overline{3 \mathrm{~Hz}}, \mathrm{CH}_{3}\right) ;{ }^{13} \mathrm{C} \mathrm{NMR}\left(125 \mathrm{MHz}, \mathrm{CDCl}_{3}\right)$ : 147.35 (Ar-C), 146.93 (Ar-C), 146.81 (Ar-C), 145.97 (Ar-C), 129.57 (Ar-C), 127.87 (Ar-C), 114.93 (Ar-C), 114.32 (Ar-C),
110.67 (Ar-C), $63.72\left(\mathrm{~d}, \mathrm{~J}_{\mathrm{PCH} 2}=7.5 \mathrm{~Hz}, \mathrm{OCH}_{2} \mathrm{CH}_{3}\right)$, $56.38\left(\mathrm{~d}, J_{\mathrm{PCH}}=157.8 \mathrm{~Hz}, \mathrm{CHP}\right), 55.64\left(\mathrm{OCH}_{3}\right), 16.87(\mathrm{~d}$, $\left.J_{\mathrm{PCH} 3}=5.0 \mathrm{~Hz}, \mathrm{CH}_{3}\right), 16.70\left(\mathrm{~d}, J_{\mathrm{PCH} 3}=5.0 \mathrm{~Hz}, \mathrm{CH}_{3}\right) ; \mathrm{MS}$ : (m/z\%), $363\left(\mathrm{M}^{+}, 21.8\right), 228$ (100), 137 (9.3).

Diethyl [(4-Nitrophenyl amino) (3,4,5-trimethoxy) methyl] phosphonate (2). This compound was synthesized after $150 \mathrm{~min}\left(72 \% . \mathrm{Mp}=89.4^{\circ} \mathrm{C}\right) .{ }^{1} \mathrm{H} \mathrm{NMR}\left(500 \mathrm{MHz}, \mathrm{CDCl}_{3}\right)$ : $8.07(\mathrm{~d}, 2 \mathrm{H}, J=6.6 \mathrm{~Hz}, \mathrm{ArH}), 6.96(\mathrm{~s}, 2 \mathrm{H}, \mathrm{ArH}), 6.64$ (s, 2H, ArH), $5.72(\mathrm{t}, 1 \mathrm{H}, J=7.1 \mathrm{~Hz}, \mathrm{NH}), 4.75(\mathrm{~d}, 1 \mathrm{H}$, $\left.J_{\mathrm{CHPO}}=23.8 \mathrm{~Hz}, \mathrm{CHP}\right), 4.12-4.21\left(\mathrm{~m}, 1 \mathrm{H}, \mathrm{OCH}_{2} \mathrm{CH}_{3}\right)$, 3.98-4.03 (m, 1H, $\left.\mathrm{OCH}_{2} \mathrm{CH}_{3}\right), 3.85\left(\mathrm{~s}, 9 \mathrm{H}, \mathrm{OCH}_{3}\right), 3.70-$ $3.75\left(\mathrm{~m}, 1 \mathrm{H}, \mathrm{OCH}_{2} \mathrm{CH}_{3}\right), 1.34\left(\mathrm{t}, 3 \mathrm{H}, J=6.6 \mathrm{~Hz}, \mathrm{CH}_{3}\right)$, $1.18\left(\mathrm{t}, 3 \mathrm{H}, J=6.6 \mathrm{~Hz}, \mathrm{CH}_{3}\right) ;{ }^{13} \mathrm{C} \mathrm{NMR}\left(125 \mathrm{MHz}, \mathrm{CDCl}_{3}\right)$ : 154.06 (Ar-C), 152.21 (Ar-C), 136.66 (Ar-C), 130.45 (ArC), 126.53 (Ar-C), 112.88 (Ar-C), 105.12 (Ar-C), 64.03 (d, $\left.{ }^{3} J_{\mathrm{PC}}=6.3 \mathrm{~Hz}, \mathrm{OCH}_{2} \mathrm{CH}_{3}\right), 59.74\left(\mathrm{~d},{ }^{3} \mathrm{~J}_{\mathrm{PC}}=7.5 \mathrm{~Hz}, \underline{\mathrm{CH}}_{3}\right)$, MS: (m/z\%), $545\left(\mathrm{M}^{+}, 5.7\right), 317$ (100), 271 (5), 181 (5.7).

Diethyl [(4-Hydroxy, 3-methoxy phenyl) (4-nitrophenyl amino) methyl]phosphonate (3). This compound was synthesized after $180 \mathrm{~min}\left(78 \% . \mathrm{Mp}=138.1^{\circ} \mathrm{C}\right) .{ }^{1} \mathrm{H}$ NMR $(500$ $\mathrm{MHz}_{\mathrm{CDCl}}$ ): 8.04 (d, 2H, $\left.J=6.6 \mathrm{~Hz}, \mathrm{ArH}\right), 7.00(\mathrm{~d}, 1 \mathrm{H}$, $J=6.6 \mathrm{~Hz}, \mathrm{ArH}), 6.97(\mathrm{~d}, 2 \mathrm{H}, J=6.6 \mathrm{~Hz}, \mathrm{ArH}), 6.95$ $(\mathrm{s}, 1 \mathrm{H}, \operatorname{ArH}), 6.64(\mathrm{~d}, 2 \mathrm{H}, J=6.6 \mathrm{~Hz}, \operatorname{ArH}), 5.86(\mathrm{t}, 1 \mathrm{H}$, $J=7.1 \mathrm{~Hz}, \mathrm{NH}), 4.76\left(\mathrm{~d}, 1 \mathrm{H}, J_{\mathrm{CHPO}}=23.3 \mathrm{~Hz}, \mathrm{CHP}\right), 4.13-$ $4.2\left(\mathrm{~m}, 2 \mathrm{H}, \mathrm{OCH}_{2} \mathrm{CH}_{3}\right), 3.95-4.00\left(\mathrm{~m}, 1 \mathrm{H}, \mathrm{OCH}_{2} \mathrm{CH}_{3}\right), 3.88$ $\left(\mathrm{s}, 3 \mathrm{H}, \mathrm{OCH}_{3}\right), 3.67-3.76\left(\mathrm{~m}, 1 \mathrm{H}, \mathrm{OCH}_{2} \mathrm{CH}_{3}\right), 1.32(\mathrm{t}, 3 \mathrm{H}$, $\left.J=6.6 \mathrm{~Hz}, \mathrm{CH}_{3}\right), 1.17\left(\mathrm{t}, 3 \mathrm{H}, J=6.6 \mathrm{~Hz}, \mathrm{CH}_{3}\right) ;{ }^{13} \mathrm{C} \mathrm{NMR}$ $\left(125 \mathrm{MHz}, \mathrm{CDCl}_{3}\right.$ ): 152.34 (Ar-C), 147.52 (Ar-C), 146.38 (Ar-C), 139.45 (Ar-C), 126.49 (Ar-C), 126.44 (Ar-C), 121.35 
TABLe 4: Antifungal activity of synthesized $\alpha$-aminophosphonates.

\begin{tabular}{|c|c|c|c|c|c|c|c|}
\hline Compound & $\begin{array}{l}\text { Candida } \\
\text { albicans }\end{array}$ & $\begin{array}{l}\text { Aspergillus } \\
\text { flavus }\end{array}$ & $\begin{array}{l}\text { Aspergillus } \\
\text { fumigatus }\end{array}$ & $\begin{array}{l}\text { Trichophyton } \\
\text { mentagrophytes }\end{array}$ & $\begin{array}{l}\text { Microsporum } \\
\text { gypsum }\end{array}$ & $\begin{array}{l}\text { Microsporum } \\
\text { canis }\end{array}$ & $\begin{array}{l}\text { Epidermophyton } \\
\text { floccosum }\end{array}$ \\
\hline \multicolumn{8}{|c|}{ MIC $\mu \mathrm{g} / \mathrm{mL}$} \\
\hline 1 & G & G & G & 2048 & 2048 & G & G \\
\hline 7 & G & G & G & 2048 & G & G & G \\
\hline 9 & G & G & G & 1024 & G & G & G \\
\hline 21 & G & G & G & G & G & 0.5 & G \\
\hline Fluconazole & 2 & 4 & 4 & NT & NT & NT & NT \\
\hline Griseofulvin & NT & NT & NT & 0.5 & 8 & 0.6 & 1 \\
\hline
\end{tabular}

G: Growth, NT: Not Tested.

(Ar-C), 115.18 (Ar-C), 112.86 (Ar-C), 110.47 (Ar-C), 64.04 $\left(\mathrm{d},{ }^{3} \mathrm{JPC}_{\mathrm{PC}}=6.3 \mathrm{~Hz}, \mathrm{OCH}_{2} \mathrm{CH}_{3}\right), 56.38\left(\mathrm{~d},{ }^{3} J_{\mathrm{PC}}=7.5 \mathrm{~Hz}\right.$, $\left.\mathrm{CH}_{3}\right), 55.07\left(\mathrm{OCH}_{3}\right) \mathrm{MS}:(\mathrm{m} / \mathrm{z} \%), 410\left(\mathrm{M}^{+}, 5.5\right), 273$ (100), 227 (8.8), 137 (7.7).

Diethyl [(3,4-Dimethoxy phenyl amino) (3,4,5-trimethoxy phenyl) methyl]phosphonate (4). This compound was synthesized after $120 \mathrm{~min}\left(87 \% . \mathrm{Mp}=105^{\circ} \mathrm{C}\right) .{ }^{1} \mathrm{H}$ NMR $\left(500 \mathrm{MHz}, \mathrm{CDCl}_{3}\right): 6.72(\mathrm{~s}, 2 \mathrm{H}, \operatorname{ArH}), 6.68(\mathrm{~d}, 1 \mathrm{H}, J=$ $6.6 \mathrm{~Hz}, \mathrm{ArH}), 6.31(\mathrm{~s}, 1 \mathrm{H}, \mathrm{ArH}), 6.11(\mathrm{~d}, 1 \mathrm{H}, J=8.3 \mathrm{~Hz}$, $\mathrm{ArH}), 4.60\left(\mathrm{~d}, 1 \mathrm{H}, J_{\mathrm{CHPO}}=23.3 \mathrm{~Hz}, \mathrm{CHP}\right), 4.11-4.19(\mathrm{~m}$, $\left.2 \mathrm{H}, \mathrm{OCH}_{2} \mathrm{CH}_{3}\right), 3.98-4.03\left(\mathrm{~m}, 1 \mathrm{H}, \mathrm{OCH}_{2} \mathrm{CH}_{3}\right), 3.84(\mathrm{~s}, 9 \mathrm{H}$, $\left.\left.\mathrm{OCH}_{3}\right), \overline{3.81}\left(\mathrm{~s}, 6 \mathrm{H}, \mathrm{OCH}_{3}\right), 3.75-3.79 \overline{(\mathrm{m}}, 1 \mathrm{H}, \mathrm{OCH}_{2} \mathrm{CH}_{3}\right)$, $1.32\left(\mathrm{t}, 3 \mathrm{H}, J=6.6 \mathrm{~Hz}, \mathrm{CH}_{3}\right), 1.18(\mathrm{t}, 3 \mathrm{H}, J=6.6 \mathrm{~Hz}$, $\left.\mathrm{CH}_{3}\right) ;{ }^{13} \mathrm{C}$ NMR $\left(125 \mathrm{MHz}, \mathrm{CDCl}_{3}\right): 153.77$ (Ar-C), 150.23 (Ar-C), 142.66 (Ar-C), 141.55 (Ar-C), 138.04 (Ar-C), 132.17 (Ar-C), 113.25 (Ar-C), 105.21 (Ar-C), 100.5 (Ar-C), 63.65 $\left(\mathrm{d}, J_{\mathrm{PC}}=6.3 \mathrm{~Hz}, \mathrm{OCH}_{2} \mathrm{CH}_{3}\right), 57.10\left(\mathrm{~d}, J_{\mathrm{PC}}=147.2 \mathrm{~Hz}\right.$ CHP), $56.91\left(\mathrm{OCH}_{3}\right), 56.58\left(\mathrm{OCH}_{3}\right), 56.1\left(\mathrm{OCH}_{3}\right), 16.88(\mathrm{~d}$, $\left.J_{\mathrm{PCH}}=5.7 \mathrm{~Hz}, \mathrm{CH}_{3}\right), 16.70\left(\mathrm{~d}, J_{\mathrm{PCH}}=5.7 \mathrm{~Hz}, \mathrm{CH}_{3}\right) ; \mathrm{MS}:$ (m/z\%), $469\left(\mathrm{M}^{+}, 7.1\right), 331$ (100), 300 (3.5), 195 (11.9).

Diethyl [(3,4-Dimethoxy phenyl) (4-nitrophenyl amino) methyl]phosphonate (5). This compound was synthesized after $180 \mathrm{~min}\left(80 \% . \mathrm{Mp}=99.2^{\circ} \mathrm{C}\right) .{ }^{1} \mathrm{H} \mathrm{NMR}\left(500 \mathrm{MHz}, \mathrm{CDCl}_{3}\right)$ : $8.04(\mathrm{~d}, 2 \mathrm{H}, J=6.6 \mathrm{~Hz}, \mathrm{ArH}), 7.01(\mathrm{~d}, 2 \mathrm{H}, J=6.6 \mathrm{~Hz}, \mathrm{ArH})$, $6.86(\mathrm{~s}, 2 \mathrm{H}, \operatorname{ArH}), 6.62(\mathrm{~d}, 2 \mathrm{H}, J=6.6 \mathrm{~Hz}, \operatorname{ArH}), 5.87(\mathrm{t}$, $1 \mathrm{H}, \mathrm{NH}), 4.74\left(\mathrm{~d}, 1 \mathrm{H}, J_{\mathrm{CHPO}}=20.01 \mathrm{~Hz}, \mathrm{CHP}\right), 4.13-4.18$ $\left(\mathrm{m}, 2 \mathrm{H}, \mathrm{OCH}_{2} \mathrm{CH}_{3}\right), 3.94-3.98\left(\mathrm{~m}, 1 \mathrm{H}, \mathrm{OCH}_{2} \mathrm{CH}_{3}\right), 3.69-$ $3.74\left(\mathrm{~m}, 1 \mathrm{H}, \mathrm{OCH}_{2} \mathrm{CH}_{3}\right), 1.32\left(\mathrm{t}, 3 \mathrm{H}, J=6.6 \mathrm{~Hz}, \mathrm{CH}_{3}\right)$, $1.16\left(\mathrm{t}, 3 \mathrm{H}, J=6.6 \mathrm{~Hz}, \mathrm{CH}_{3}\right) ;{ }^{13} \mathrm{C} \mathrm{NMR}\left(125 \mathrm{MHz}, \mathrm{CDCl}_{3}\right)$ : 152.35 (Ar-C), 149.78 (Ar-C), 149.60 (Ar-C), 139.45 (Ar-C), 127.15 (Ar-C), 126.48 (Ar-C), 120.63 (Ar-C), 112.86 (ArC), $111.67(\mathrm{Ar}-\mathrm{C}), 111.08(\mathrm{Ar}-\mathrm{C}), 63.98\left(\mathrm{~d}, J_{\mathrm{PC}}=7.5 \mathrm{~Hz}\right.$, $\left.\mathrm{OCH}_{2} \mathrm{CH}_{3}\right), 56.32\left(\mathrm{~d}, J_{\mathrm{PC}}=149.2 \mathrm{~Hz} \mathrm{CHP}\right), 55.03\left(\mathrm{OCH}_{3}\right)$, $56.58\left(\mathrm{OCH}_{3}\right), 16.85\left(\mathrm{~d}, J_{\mathrm{PCH}}=7.5 \mathrm{~Hz}, \mathrm{CH}_{3}\right), 16.70(\mathrm{~d}$, $\left.J_{\mathrm{PCH}}=7.5 \mathrm{~Hz}, \mathrm{CH}_{3}\right) ; \mathrm{MS}:\left(\mathrm{m} / \mathrm{z}^{2}\right), 424\left(\mathrm{M}^{+}, 5.5\right), 287(100)$, 241 (7), 149 (2.9).

Diethyl [(5-Chloro-2-methylphenyl amino) (3,4,5-trimethoxy phenyl) methyl]phosphonate (6). This compound was synthesized after $120 \mathrm{~min}\left(76 \% \mathrm{Mp}=107^{\circ} \mathrm{C}\right) .{ }^{1} \mathrm{H} \mathrm{NMR}$ $\left(500 \mathrm{MHz}, \mathrm{CDCl}_{3}\right): 6.96(\mathrm{~s}, 1 \mathrm{H}, \operatorname{ArH}), 6.94(\mathrm{~d}, 1 \mathrm{H}, J=$ $8.3 \mathrm{~Hz}, \mathrm{ArH}), 6.7$ (s, 2H, ArH), $6.45(\mathrm{~s}, 1 \mathrm{H}, \mathrm{ArH}), 4.67$ $\left(\mathrm{d}, 1 \mathrm{H}, J_{\mathrm{CHPO}}=26.60 \mathrm{~Hz}, \mathrm{CHP}\right), 4.09-4.17(\mathrm{~m}, 2 \mathrm{H}$, $\left.\mathrm{OCH}_{2} \mathrm{CH}_{3}\right), 3.96-4.01\left(\mathrm{~m}, 1 \mathrm{H}, \mathrm{OCH}_{2} \mathrm{CH}_{3}\right), 3.85(\mathrm{~s}, 9 \mathrm{H}$, $\left.\mathrm{OC}_{\overline{\mathrm{H}}}\right), 3.72-3.77\left(\mathrm{~m}, 1 \mathrm{H}, \mathrm{OCH}_{2} \mathrm{CH}_{3}\right), 2.24\left(\mathrm{~s}, 3 \mathrm{H}, \mathrm{CH}_{3}\right)$, $1.29\left(\mathrm{t}, 3 \mathrm{H}, J=6.6 \mathrm{~Hz}, \mathrm{CH}_{3}\right), 1.16\left(\mathrm{t}, 3 \mathrm{H}, J=6.6 \mathrm{~Hz}, \mathrm{CH}_{3}\right)$; ${ }^{13} \mathrm{C}$ NMR (125 MHz, $\mathrm{CDCl}_{3}$ ): 153.86 (Ar-C), 145.98 (Ar-C), 138.28 (Ar-C), 132.85 (Ar-C), 131.41 (Ar-C), 121.55 (ArC), $118.32(\mathrm{Ar}-\mathrm{C}), 111.78(\mathrm{Ar}-\mathrm{C}), 105.14(\mathrm{Ar}-\mathrm{C}), 63.76(\mathrm{~d}$, $\left.J_{\mathrm{PC}}=6.3 \mathrm{~Hz}, \mathrm{OCH}_{2} \mathrm{CH}_{3}\right), 57.30\left(\mathrm{~d}, J_{\mathrm{PC}}=147.2 \mathrm{~Hz} \mathrm{CHP}\right)$, $56.60\left(\mathrm{OCH}_{3}\right), 17.47(\mathrm{CH} 3), 16.84\left(\mathrm{~d}, J_{\mathrm{PCH}}=7.5 \mathrm{~Hz}, \mathrm{CH}_{3}\right)$, $16.66\left(\mathrm{~d}, J_{\mathrm{PCH}}=7.5 \mathrm{~Hz}, \mathrm{CH}_{3}\right) ; \mathrm{MS}:(\mathrm{m} / \mathrm{z} \%), 457\left(\mathrm{M}^{+}, 5.3\right)$, 320 (100), 181 (6.6).

Diethyl [(5-Chloro-2-methylphenyl amino) (4-methoxy phenyl) methyl]phosphonate (7). This compound was synthesized after $120 \mathrm{~min}\left(77 \% . \mathrm{Mp}=102.4^{\circ} \mathrm{C}\right) .{ }^{1} \mathrm{H}$ NMR $\left(500 \mathrm{MHz}, \mathrm{CDCl}_{3}\right): 7.40(\mathrm{~d}, 2 \mathrm{H}, J=6.6 \mathrm{~Hz}, \mathrm{ArH}), 6.97$ (s, $1 \mathrm{H}, \operatorname{ArH}), 6.92(\mathrm{~d}, 2 \mathrm{H}, J=6.6 \mathrm{~Hz}, \mathrm{ArH}), 6.64(\mathrm{~d}, 1 \mathrm{H}$, $J=6.6 \mathrm{~Hz}, \mathrm{ArH}), 6.43(\mathrm{~d}, 1 \mathrm{H}, J=6.6 \mathrm{~Hz}, \mathrm{ArH}), 4.71(\mathrm{~d}, 1 \mathrm{H}$, $\left.J_{\mathrm{CHPO}}=23.30 \mathrm{~Hz}, \mathrm{CHP}\right), 4.11-4.19\left(\mathrm{~m}, 2 \mathrm{H}, \mathrm{OCH}_{2} \mathrm{CH}_{3}\right)$, $3.95-4.00\left(\mathrm{~m}, 1 \mathrm{H}, \mathrm{OCH}_{2} \mathrm{CH}_{3}\right), 3.82\left(\mathrm{~s}, 3 \mathrm{H}, \mathrm{OCH}_{3}\right), 3.71-$ $3.76\left(\mathrm{~m}, 1 \mathrm{H}, \mathrm{OCH}_{2} \mathrm{CH}_{3}\right), 2.25\left(\mathrm{~s}, 3 \mathrm{H}, \mathrm{CH}_{3}\right), 1.32(\mathrm{t}, 3 \mathrm{H}$, $\left.J=6.6 \mathrm{~Hz}, \mathrm{CH}_{3}\right), 1.17\left(\mathrm{t}, 3 \mathrm{H}, J=6.6 \mathrm{~Hz}, \mathrm{CH}_{3}\right) ;{ }^{13} \mathrm{C} \mathrm{NMR}$ $\left(125 \mathrm{MHz}, \mathrm{CDCl}_{3}\right): 159.88$ (Ar-C), 145.85 (Ar-C), 132.83 (Ar-C), 131.35 (Ar-C), 129.19 (Ar-C), 127.54 (Ar-C), 121.59 (Ar-C), 118.10 (Ar-C), 114.63 (Ar-C), 111.71 (Ar-C), 63.68 $\left(\mathrm{d}, J_{\mathrm{PC}}=3.75 \mathrm{~Hz}, \mathrm{OCH}_{2} \mathrm{CH}_{3}\right), 56.28\left(\mathrm{~d}, J_{\mathrm{PC}}=152 \mathrm{~Hz} \mathrm{CHP}\right)$, $55.66\left(\mathrm{OCH}_{3}\right), 17.48(\mathrm{CH} 3), 16.86\left(\mathrm{~d}, \mathrm{~J}_{\mathrm{PCH}}=5.75 \mathrm{~Hz}, \mathrm{CH}_{3}\right)$, $16.68\left(\mathrm{~d}, J_{\mathrm{PCH}}=5.75 \mathrm{~Hz}, \mathrm{CH}_{3}\right) ; \mathrm{MS}:(\mathrm{m} / \mathrm{z} \%), 397\left(\mathrm{M}^{+}, 7.0\right)$, 260 (100), 121 (17.8).

Diethyl [(3,4-Dimethoxyphenyl) (phenyl amino) methyl]phosphonate (8). This compound was synthesized after $120 \mathrm{~min}$ $\left(82 \% . \mathrm{Mp}=103^{\circ} \mathrm{C}\right) .{ }^{1} \mathrm{H}$ NMR $\left(500 \mathrm{MHz}, \mathrm{CDCl}_{3}\right): 7.13(\mathrm{t}$, $2 \mathrm{H}, J=8.3 \mathrm{~Hz}, \mathrm{ArH}), 7.04(\mathrm{~d}, 2 \mathrm{H}, J=8.3 \mathrm{~Hz}, \mathrm{ArH}), 6.85$ $(\mathrm{s}, 1 \mathrm{H}, \operatorname{ArH}), 6.72(\mathrm{t}, 1 \mathrm{H}, J=8.3 \mathrm{~Hz}, \mathrm{ArH}), 6.63(\mathrm{~d}, 2 \mathrm{H}, J=$ $8.3 \mathrm{~Hz}, \mathrm{ArH}), 4.72\left(\mathrm{~d}, 1 \mathrm{H}, J_{\mathrm{CHPO}}=18.30 \mathrm{~Hz}, \mathrm{CHP}\right), 4.10$ $4.17\left(\mathrm{~m}, 2 \mathrm{H}, \mathrm{OCH}_{2} \mathrm{CH}_{3}\right), 3.96-4.01\left(\mathrm{~m}, 1 \mathrm{H}, \mathrm{OCH}_{2} \mathrm{CH}_{3}\right)$, $3.88\left(\mathrm{~s}, 6 \mathrm{H}, \mathrm{OCH}_{3}\right), 3.71-3.76\left(\mathrm{~m}, 1 \mathrm{H}, \mathrm{OCH}_{2} \mathrm{CH}_{3}\right), 1.31$ $\left(\mathrm{t}, 3 \mathrm{H}, J=8.3 \mathrm{~Hz}, \mathrm{CH}_{3}\right), 1.17\left(\mathrm{t}, 3 \mathrm{H}, J=8.3 \mathrm{~Hz}, \mathrm{CH}_{3}\right)$; ${ }^{13} \mathrm{C}$ NMR (125 MHz, $\mathrm{CDCl}_{3}$ ): 149.52 (Ar-C), 149.14 (Ar-C), 146.87 (Ar-C), 129.56 (Ar-C), 128.65 (Ar-C), 120.63 (Ar-C), 118.84 (Ar-C), 114.31 (Ar-C), 111.54 (Ar-C), 111.25 (ArC), $63.64\left(\mathrm{~d}, J_{\mathrm{PC}}=4.60 \mathrm{~Hz}, \mathrm{OCH}_{2} \mathrm{CH}_{3}\right), 56.83\left(\mathrm{~d}, J_{\mathrm{PC}}=\right.$ $187.25 \mathrm{~Hz} \mathrm{CHP}), 56.30\left(\mathrm{OCH}_{3}\right), 16.88\left(\mathrm{~d}, J_{\mathrm{PCH}}=5.80 \mathrm{~Hz}\right.$, 
$\left.\mathrm{CH}_{3}\right), 16.72\left(\mathrm{~d}, J_{\mathrm{PCH}}=5.80 \mathrm{~Hz}, \mathrm{CH}_{3}\right) ; \mathrm{MS}:(\mathrm{m} / \mathrm{z} \%), 379$ $\left(\mathrm{M}^{+}, 5.0\right), 242$ (100), 151 (17.8).

Diethyl [(4-Chloro-2-nitrophenyl amino) (4-hydroxy-3-methoxyphenyl) methyl]phosphonate (9). This compound was synthesized after $180 \mathrm{~min}\left(70 \% . \mathrm{Mp}=187.4^{\circ} \mathrm{C}\right) .{ }^{1} \mathrm{H}$ NMR $\left(500 \mathrm{MHz}, \mathrm{CDCl}_{3}\right): 8.22(\mathrm{~s}, 1 \mathrm{H}, \mathrm{ArH}), 7.30(\mathrm{~d}, 1 \mathrm{H}, J=$ $6.6 \mathrm{~Hz}, \mathrm{ArH}), 6.99(\mathrm{~d}, 1 \mathrm{H}, J=6.6 \mathrm{~Hz}, \mathrm{ArH}), 6.93(\mathrm{~d}, 2 \mathrm{H}$, $J=6.6 \mathrm{~Hz}, \operatorname{ArH}), 6.71(\mathrm{~s}, 1 \mathrm{H}, \mathrm{ArH}), 4.82\left(\mathrm{~d}, 1 \mathrm{H}, J_{\mathrm{CHPO}}=\right.$ $23.8 \mathrm{~Hz}, \mathrm{CHP}), 4.03-4.14\left(\mathrm{~m}, 2 \mathrm{H}, \mathrm{OCH}_{2} \mathrm{CH}_{3}\right), 3.96-3.99$ $\left(\mathrm{m}, 1 \mathrm{H}, \mathrm{OCH}_{2} \mathrm{CH}_{3}\right), 3.91\left(\mathrm{~s}, 3 \mathrm{H}, \mathrm{OCH}_{3}\right), 3.71-3.76(\mathrm{~m}, 1 \mathrm{H}$, $\left.\mathrm{OCH}_{2} \mathrm{CH}_{3}\right), 1.28\left(\mathrm{t}, 3 \mathrm{H}, J=6.6 \mathrm{~Hz}, \mathrm{CH}_{3}\right), 1.17(\mathrm{t}, 3 \mathrm{H}$, $\left.J=6.6 \mathrm{~Hz}, \mathrm{CH}_{3}\right) ;{ }^{13} \mathrm{C} \mathrm{NMR}\left(125 \mathrm{MHz}, \mathrm{CDCl}_{3}\right): 147.53(\mathrm{Ar}-$ C), 146.42 (Ar-C), 143.15 (Ar-C), 136.59 (Ar-C), 133.52 (ArC), 126.41 (Ar-C), 126.03 (Ar-C), 122.01 (Ar-C), 121.05 (ArC), $116.66(\mathrm{Ar}-\mathrm{C}), 115.14(\mathrm{Ar}-\mathrm{C}), 110.21(\mathrm{Ar}-\mathrm{C}), 64.11(\mathrm{~d}$, $\left.J_{\mathrm{PC}}=8.0 \mathrm{~Hz}, \mathrm{OCH}_{2} \mathrm{CH}_{3}\right), 56.46\left(\mathrm{~d}, J_{\mathrm{PC}}=150.9 \mathrm{~Hz} \mathrm{CHP}\right)$, $55.23\left(\mathrm{OCH}_{3}\right), 16.84\left(\mathrm{~d}, J_{\mathrm{PCH}}=5.9 \mathrm{~Hz}, \mathrm{CH}_{3}\right), 16.75(\mathrm{~d}$, $\left.J_{\mathrm{PCH}}=5.9 \mathrm{~Hz}, \mathrm{CH}_{3}\right) ; \mathrm{MS}:\left(\mathrm{m} / \mathrm{z}^{2}\right), 444\left(\mathrm{M}^{+}, 1.0\right), 307(100)$, 290 (23.5), 273 (41.1), 151 (26.4).

Diethyl [(3,4-Dimethoxy phenyl amino) (4-hydroxy-3-methoxy phenyl) methyl]phosphonate (10). This compound was synthesized after $120 \mathrm{~min}\left(81 \% . \mathrm{Mp}=103.2^{\circ} \mathrm{C}\right) .{ }^{1} \mathrm{H}$ NMR $\left(500 \mathrm{MHz}, \mathrm{CDCl}_{3}\right): 7.00(\mathrm{~s}, 2 \mathrm{H}, \operatorname{ArH}), 6.81(\mathrm{~d}, 1 \mathrm{H}, J=$ $6.6 \mathrm{~Hz}, \mathrm{ArH}), 6.64(\mathrm{~d}, 1 \mathrm{H}, J=6.6 \mathrm{~Hz}, \mathrm{ArH}), 6.26(\mathrm{~d}, 1 \mathrm{H}$, $J=6.6 \mathrm{~Hz}, \mathrm{ArH}), 6.09(\mathrm{~d}, 1 \mathrm{H}, J=6.6 \mathrm{~Hz}, \operatorname{ArH}), 4.60(\mathrm{~d}$, $\left.1 \mathrm{H}, J_{\mathrm{CHPO}}=26.0 \mathrm{~Hz}, \mathrm{CHP}\right), 4.07-4.16\left(\mathrm{~m}, 2 \mathrm{H}, \mathrm{OCH}_{2} \mathrm{CH}_{3}\right)$, $3.93-3.98\left(\mathrm{~m}, 1 \mathrm{H}, \mathrm{OCH}_{2} \mathrm{CH}_{3}\right), 3.85\left(\mathrm{~s}, 6 \mathrm{H}, \mathrm{OCH}_{3}\right), 3.75$ $\left(\mathrm{s}, 3 \mathrm{H}, \mathrm{OCH}_{3}\right), 3.69-3 . \overline{73}\left(\mathrm{~m}, 1 \mathrm{H}, \mathrm{OCH}_{2} \mathrm{CH}_{3}\right), 1.29(\mathrm{t}, 3 \mathrm{H}$, $\left.J=8.3 \mathrm{~Hz}, \mathrm{CH}_{3}\right), 1.14\left(\mathrm{t}, 3 \mathrm{H}, J=8.3 \mathrm{~Hz}, \mathrm{CH}_{3}\right) ;{ }^{13} \mathrm{C}$ NMR (125 MHz, $\left.\mathrm{CDCl}_{3}\right)$ : 150.20 (Ar-C), 149.50 (Ar-C), 142.53 (Ar-C), 141.61 (Ar-C), 141.48 (Ar-C), 128.82 (Ar-C), 120.61 (Ar-C), 113.27 (Ar-C), 111.50 (Ar-C), 111.26 (ArC), 105.19 (Ar-C), 100.52(Ar-C), $63.60\left(\mathrm{~d}, J_{\mathrm{PC}}=6.3 \mathrm{~Hz}\right.$, $\left.\mathrm{OCH}_{2} \mathrm{CH}_{3}\right), 56.90\left(\mathrm{~d}, J_{\mathrm{PC}}=147.2 \mathrm{~Hz} \mathrm{CHP}\right), 56.32\left(\mathrm{OCH}_{3}\right)$, $56.05\left(\mathrm{OCH}_{3}\right), 16.86\left(\mathrm{~d}, J_{\mathrm{PCH}}=5.7 \mathrm{~Hz}, \mathrm{CH}_{3}\right), 16.70(\mathrm{~d}$, $\left.J_{\mathrm{PCH}}=5.7 \mathrm{~Hz}, \mathrm{CH}_{3}\right) ; \mathrm{MS}:(\mathrm{m} / \mathrm{z} \%), 425\left(\mathrm{M}^{+}, 25\right), 288(100)$, $272(3.5), 1490(8.3)$.

Diethyl [(Phenyl amino) (3,4,5-trimethoxy phenyl) methyl] phosphonate (11). This compound was synthesized after 30 $\min \left(81 \% . \mathrm{Mp}=109^{\circ} \mathrm{C}\right) .{ }^{1} \mathrm{H}$ NMR $\left(500 \mathrm{MHz}, \mathrm{CDCl}_{3}\right)$ : $7.16(\mathrm{t}, 2 \mathrm{H}, J=7.25 \mathrm{~Hz}, \mathrm{ArH}), 6.75(\mathrm{t}, 3 \mathrm{H}, J=7.3 \mathrm{ArH})$, $6.65(\mathrm{~d}, 2 \mathrm{H}, J=7.56 \mathrm{~Hz}, \operatorname{ArH}), 4.80\left(\mathrm{~d}, 1 \mathrm{H}, J_{\mathrm{CHPO}}=\right.$ $41.7 \mathrm{~Hz}, \mathrm{CHP}), 4.10-4.17\left(\mathrm{~m}, 2 \mathrm{H}, \mathrm{OCH}_{2} \mathrm{CH}_{3}\right), 3.99-4.03$ $\left(\mathrm{m}, 1 \mathrm{H}, \mathrm{OCH}_{2} \mathrm{CH}_{3}\right), 3.85\left(\mathrm{~s}, 9 \mathrm{H}, \mathrm{OCH}_{3}\right), 3.75-3.79(\mathrm{~m}, 1 \mathrm{H}$, $\left.\mathrm{OCH}_{2} \mathrm{CH}_{3}\right), 1.32\left(\mathrm{t}, 3 \mathrm{H}, J=7.3 \mathrm{~Hz}, \mathrm{CH}_{3}\right), 1.18(\mathrm{t}, 3 \mathrm{H}$, $\left.J=7.3 \mathrm{~Hz}, \mathrm{CH}_{3}\right) ;{ }^{13} \mathrm{C} \mathrm{NMR}\left(125 \mathrm{MHz}, \mathrm{CDCl}_{3}\right): 153.76(\mathrm{Ar}-$ C), 146.92 (Ar-C), 131.96 ( $\mathrm{Ar}-\mathrm{C}), 129.61$ (Ar-C), 118.96 (ArC), 114.27 (Ar-C), $105.23(\mathrm{Ar}-\mathrm{C}), 63.72\left(\mathrm{~d}, J_{\mathrm{PC}}=5.1 \mathrm{~Hz}\right.$, $\left.\mathrm{OCH}_{2} \mathrm{CH}_{3}\right), 56.81\left(\mathrm{~d}, J_{\mathrm{PC}}=149 \mathrm{~Hz} \mathrm{CHP}\right), 56.57\left(\mathrm{OCH}_{3}\right)$, $56.21\left(\mathrm{OCH}_{3}\right), 16.87\left(\mathrm{~d}, J_{\mathrm{PCH}}=5.6 \mathrm{~Hz}, \mathrm{CH}_{3}\right), 16.70(\mathrm{~d}$, $\left.J_{\mathrm{PCH}}=6.0 \mathrm{~Hz}, \mathrm{CH}_{3}\right) ; \mathrm{MS}:(\mathrm{m} / \mathrm{z} \%), 409\left(\mathrm{M}^{+}, 7.4\right), 272(100)$, $181(5.4)$.

Diethyl [4-Methoxyphenyl)(phenyl amino)methyl]phosphonate (12). This compound was synthesized after $60 \mathrm{~min}(76 \%$.
$\left.\mathrm{Mp}=102^{\circ} \mathrm{C}\right) .{ }^{1} \mathrm{H}$ NMR $\left(500 \mathrm{MHz}, \mathrm{CDCl}_{3}\right): 7.42(\mathrm{~d}, 2 \mathrm{H}$, $J=7.5 \mathrm{~Hz}, \operatorname{ArH}), 7.13(\mathrm{t}, 2 \mathrm{H}, J=7.5 \mathrm{~Hz}, \operatorname{ArH}), 6.90(\mathrm{~d}$, $2 \mathrm{H} J=8.5 \mathrm{~Hz}, \mathrm{ArH}), 6.72(\mathrm{~d}, 2 \mathrm{H}, J=8.5 \mathrm{~Hz}, \mathrm{ArH}), 6.72$ $(\mathrm{t}, 1 \mathrm{H}, J=7.25 \mathrm{~Hz}, \operatorname{ArH}), 6.63(\mathrm{~d}, 2 \mathrm{H}, J=8.2 \mathrm{~Hz}, \operatorname{ArH})$, $4.78(\mathrm{~s}, 1 \mathrm{H}, \mathrm{NH}), 4.76\left(\mathrm{~d}, 1 \mathrm{H}, J_{\mathrm{CHPO}}=40.1 \mathrm{~Hz}, \mathrm{CHP}\right), 4.11-$ $4.17\left(\mathrm{~m}, 2 \mathrm{H}, \mathrm{OCH}_{2} \mathrm{CH}_{3}\right), 3.96-4.0\left(\mathrm{~m}, 1 \mathrm{H}, \mathrm{OCH}_{2} \mathrm{CH}_{3}\right)$, $3.80\left(\mathrm{~s}, 3 \mathrm{H}, \mathrm{OCH}_{3}\right), 3.71-3.76\left(\mathrm{~m}, 1 \mathrm{H}, \mathrm{OCH}_{2} \mathrm{CH}_{3}\right), 1.32$ $\left(\mathrm{t}, 3 \mathrm{H}, J=7.3 \mathrm{~Hz}, \mathrm{CH}_{3}\right), 1.17\left(\mathrm{t}, 3 \mathrm{H}, J=\overline{7.3} \mathrm{~Hz}, \mathrm{CH}_{3}\right)$; ${ }^{13} \mathrm{C}$ NMR (125 MHz, $\left.\mathrm{CDCl}_{3}\right)$ : 159.74 (Ar-C), 146.79 (ArC), 129.57 (Ar-C), 129.38 (Ar-C), 128.11 (Ar-C), 118.76 (ArC), $114.47(\mathrm{Ar}-\mathrm{C}), 114.31(\mathrm{Ar}-\mathrm{C}), 63.62\left(\mathrm{~d},{ }^{3} J_{\mathrm{PC}}=4.75 \mathrm{~Hz}\right.$, $\left.\mathrm{OCH}_{2} \mathrm{CH}_{3}\right), 55.87\left(\mathrm{~d},{ }^{3} \mathrm{~J}_{\mathrm{PC}}=151 \mathrm{~Hz}, \mathrm{CHP}\right), 55.64\left(\mathrm{OCH}_{3}\right)$, $16.85\left(\mathrm{~d}, J_{\mathrm{PCH} 3}=5.7 \mathrm{~Hz}, \mathrm{CH}_{3}\right), 16.68\left(\mathrm{~d}, J_{\mathrm{PCH} 3}=5.7 \mathrm{~Hz}\right.$, $\left.\mathrm{CH}_{3}\right)$; MS: (m/z\%), $349\left(\mathrm{M}^{+}, 3.9\right), 212$ (100), 121 (3.9).

Diethyl [(4-Chloro-2-nitrophenyl amino) (3,4,5-trimethoxy phenyl) methyl]phosphonate (13). This compound was synthesized after $90 \mathrm{~min}\left(75 \% . \mathrm{Mp}=137^{\circ} \mathrm{C}\right) .{ }^{1} \mathrm{H}$ NMR $\left(500 \mathrm{MHz}, \mathrm{CDCl}_{3}\right): 8.84(\mathrm{~d}, 1 \mathrm{H}, J=11.0 \mathrm{~Hz}, \mathrm{ArH}), 8.19$ (s, $1 \mathrm{H}, \operatorname{ArH}), 6.67-677(\mathrm{~m}, 3 \mathrm{H}, \operatorname{ArH}), 4.79\left(\mathrm{~d}, 1 \mathrm{H}, J_{\mathrm{CHPO}}=\right.$ $23.25 \mathrm{~Hz}, \mathrm{CHP}), 4.09-4.14\left(\mathrm{~m}, 2 \mathrm{H}, \mathrm{OCH}_{2} \mathrm{CH}_{3}\right), 4.03-4.07$ $\left(\mathrm{m}, 1 \mathrm{H}, \mathrm{OCH}_{2} \mathrm{CH}_{3}\right), 3.97-3.99\left(\mathrm{~m}, 1 \mathrm{H}, \overline{\mathrm{OC}} \mathrm{H}_{2} \mathrm{CH}_{3}\right), 3.84(\mathrm{~s}$, $\left.9 \mathrm{H}, \mathrm{OCH}_{3}\right), 1.31-1.25\left(\mathrm{~m}, 6 \mathrm{H}, J=7.3 \mathrm{~Hz}, \mathrm{CH}_{3}\right) ;{ }^{13} \mathrm{C} \mathrm{NMR}$ (125 MHz, $\left.\mathrm{CDCl}_{3}\right)$ : 154.6 (Ar-C), 143.17 (Ar-C), $143.06(\mathrm{Ar}-$ C), 138.53 (Ar-C), 136.66 (Ar-C), 130.09 (Ar-C), 130.06 (Ar-C), 126.38 (Ar-C), 122.15 (Ar-C), 116.67 (Ar-C), 64.19 $\left(\mathrm{d}, J_{\mathrm{PC}}=7.1 \mathrm{~Hz}, \mathrm{OCH}_{2} \mathrm{CH}_{3}\right), 64.02\left(\mathrm{~d}, J_{\mathrm{PC}}=7.25 \mathrm{~Hz}\right.$, $\left.\mathrm{OCH}_{2} \mathrm{CH}_{3}\right), 56.66\left(\mathrm{OCH}_{3}\right), 56.32\left(\mathrm{~d}, J_{\mathrm{PC}}=151 \mathrm{~Hz} \mathrm{CHP}\right)$, $16.82\left(\mathrm{~d}, J_{\mathrm{PCH}}=5.7 \mathrm{~Hz}, \mathrm{CH}_{3}\right), 16.73\left(\mathrm{~d}, J_{\mathrm{PCH}}=5.70 \mathrm{~Hz}\right.$, $\left.\mathrm{CH}_{3}\right)$; MS: (m/z\%), $488\left(\mathrm{M}^{+}, 4.3\right), 351$ (100), 195 (8.7).

Diethyl [(Phenyl amino)(phenyl)methyl]phosphonate (14). This compound was synthesized after $90 \mathrm{~min}(73 \%$. $\mathrm{Mp}=$ $\left.77^{\circ} \mathrm{C}\right) .{ }^{1} \mathrm{H}$ NMR $\left(500 \mathrm{MHz}, \mathrm{CDCl}_{3}\right): 7.53$ (s, $\left.2 \mathrm{H}, \mathrm{ArH}\right), 7.38$ $(\mathrm{t}, 2 \mathrm{H}, J=7.5 \mathrm{~Hz}, \mathrm{ArH}), 7.29-7.32(\mathrm{~m}, 1 \mathrm{H}, \mathrm{ArH}), 7.15(\mathrm{t}$, $2 \mathrm{H}, J=8.2 \mathrm{~Hz}, \mathrm{ArH}), 6.74(\mathrm{t}, 1 \mathrm{H}, J=7.5 \mathrm{~Hz}, \mathrm{ArH}), 6.65$ $(\mathrm{d}, 2 \mathrm{H}, J=8.5 \mathrm{~Hz}, \mathrm{ArH}), 4.82\left(\mathrm{~d}, 1 \mathrm{H}, J_{\mathrm{CHPO}}=24.57 \mathrm{~Hz}\right.$, CHP), 4.11-4.21 (m, 2H, OCH $\left.\mathrm{CH}_{3}\right), 3.96-4.01(\mathrm{~m}, 1 \mathrm{H}$, $\left.\mathrm{OCH}_{2} \mathrm{CH}_{3}\right), 3.70-3.75\left(\mathrm{~m}, 1 \overline{\mathrm{H},} \mathrm{OCH}_{2} \mathrm{CH}_{3}\right), 1.33(\mathrm{t}, 3 \mathrm{H}$, $\left.J=7.0 \mathrm{~Hz}, \mathrm{CH}_{3}\right), 1.16\left(\mathrm{t}, 3 \mathrm{H}, J=7.3 \mathrm{~Hz}, \mathrm{CH}_{3}\right) ;{ }^{13} \mathrm{C} \mathrm{NMR}$ $\left(125 \mathrm{MHz}, \mathrm{CDCl}_{3}\right): 146.80$ (Ar-C), 136.35 (Ar-C), 129.60 (Ar-C), 129.02 (Ar-C), 128.34 (Ar-C), 128.30 (Ar-C), 118.82 $(\mathrm{Ar}-\mathrm{C}), 114.29(\mathrm{Ar}-\mathrm{C}), 63.72\left(\mathrm{~d}, J_{\mathrm{PC}}=4.8 \mathrm{~Hz}, \mathrm{OCH}_{2} \mathrm{CH}_{3}\right)$, $63.66\left(\mathrm{~d}, J_{\mathrm{PC}}=4.8 \mathrm{~Hz}, \mathrm{OCH}_{2} \mathrm{CH}_{3}\right), 56.50\left(\mathrm{~d}, J_{\mathrm{PC}}=149 \mathrm{~Hz}\right.$, $\mathrm{CHP}), 16.88\left(\mathrm{~d}, J_{\mathrm{PCH} 3}=5.7 \mathrm{~Hz}, \mathrm{CH}_{3}\right), 16.64\left(\mathrm{~d}, J_{\mathrm{PCH} 3}=\right.$ $\left.5.8 \mathrm{~Hz}, \mathrm{CH}_{3}\right)$; MS: (m/z\%), $319\left(\mathrm{M}^{+}, 16\right), 182(100)$.

Diethyl [(3,4-Dimethoxy phenyl amino) (3,4-dimethoxy phenyl) methyl]phosphonate (15). This compound was synthesized after $45 \mathrm{~min}\left(75 \% \mathrm{Mp}=115^{\circ} \mathrm{C}\right) .{ }^{1} \mathrm{H}$ NMR $(500 \mathrm{MHz}$, $\left.\mathrm{CDCl}_{3}\right)$ : 6.99-7.01 (m, $\left.2 \mathrm{H}, \mathrm{ArH}\right), 6.82(\mathrm{~d}, 1 \mathrm{H}, J=8.1 \mathrm{~Hz}$, $\mathrm{ArH}), 6.63(\mathrm{~d}, 1 \mathrm{H}, J=8.5 \mathrm{~Hz}, \mathrm{ArH}), 6.29(\mathrm{~s}, 1 \mathrm{H}, \mathrm{ArH})$, $6.08(\mathrm{~d}, 1 \mathrm{H}, J=7.1 \mathrm{~Hz}, \mathrm{ArH}), 4.65(\mathrm{~s}, 1 \mathrm{H}, \mathrm{NH}), 4.59(\mathrm{~d}$, $\left.1 \mathrm{H}, J_{\mathrm{CHPO}}=23.3 \mathrm{~Hz}, \mathrm{CHP}\right), 4.07-4.16\left(\mathrm{~m}, 2 \mathrm{H}, \mathrm{OCH}_{2} \mathrm{CH}_{3}\right)$, 3.93-3.98 (m, $\left.1 \mathrm{H}, \mathrm{OCH}_{2} \mathrm{CH}_{3}\right), 3.85\left(\mathrm{~s}, 6 \mathrm{H}, \mathrm{OCH}_{3}\right), 3.74$ $\left(\mathrm{s}, 6 \mathrm{H}, \mathrm{OCH}_{3}\right), 3.69-3.74\left(\mathrm{~m}, 1 \mathrm{H}, \mathrm{OCH}_{2} \mathrm{CH}_{3}\right), 1.29(\mathrm{t}, 3 \mathrm{H}$, $\left.J=6.6 \mathrm{~Hz}, \mathrm{CH}_{3}\right), 1.14\left(\mathrm{t}, 3 \mathrm{H}, J=6.6 \mathrm{~Hz}, \mathrm{CH}_{3}\right) ;{ }^{13} \mathrm{C} \mathrm{NMR}$ $\left(125 \mathrm{MHz}, \mathrm{CDCl}_{3}\right)$ : 150.20 (Ar-C), 149.50 (Ar-C), 149.10 
(Ar-C), 142.53 (Ar-C), 141.55 (Ar-C), 128.82 (Ar-C), 120.63 (Ar-C), 113.27 (Ar-C), 111.50 (Ar-C), 111.26 (Ar-C), 105.19 $(\mathrm{Ar}-\mathrm{C}), 100.52(\mathrm{Ar}-\mathrm{C}), 63.63\left(\mathrm{~d}, J_{\mathrm{PC}}=3.0 \mathrm{~Hz}, \mathrm{OCH}_{2} \mathrm{CH}_{3}\right)$, $63.58\left(\mathrm{~d}, J_{\mathrm{PC}}=3.0 \mathrm{~Hz}, \mathrm{OCH}_{2} \mathrm{CH}_{3}\right), 57.03\left(\mathrm{~d}, J_{\mathrm{PC}}=151.0 \mathrm{~Hz}\right.$ $\mathrm{CHP}), 56.99\left(\mathrm{OCH}_{3}\right), 56.32\left(\mathrm{OCH}_{3}\right), 56.23\left(\mathrm{OCH}_{3}\right), 56.05$ $\left(\mathrm{OCH}_{3}\right), 16.86\left(\mathrm{~d}, J_{\mathrm{PCH}}=5.6 \mathrm{~Hz}, \mathrm{CH}_{3}\right), 16.70\left(\mathrm{~d}, J_{\mathrm{PCH}}=\right.$ $\left.5.6 \mathrm{~Hz}, \mathrm{CH}_{3}\right)$; MS: (m/z\%), $439\left(\mathrm{M}^{+}, 9.3\right), 302$ (100), 151 (6.6).

Diethyl [(5-Chloro-2-methylphenyl amino) (4-hydroxy-3-methoxy phenyl) methyl]phosphonate (16). This compound was synthesized after $90 \mathrm{~min}(64 \% . \mathrm{Mp}=152.4 \circ \mathrm{C}) .1 \mathrm{H} \mathrm{NMR}$ (500 MHz, CDCl3): 7.01 (s, 1H, ArH), 6.95-6.98 (m, 2H, ArH), 6.91 (s, 1H, ArH), $6.65(\mathrm{~d}, 1 \mathrm{H}, J=6.1 \mathrm{~Hz}, \mathrm{ArH}), 6.46$ (s, 1H, ArH), $4.71(\mathrm{~s}, 1 \mathrm{H}, \mathrm{CHP}), 4.66(\mathrm{~s}, 1 \mathrm{H}, \mathrm{NH}), 4.09-$ $4.19(\mathrm{~m}, 2 \mathrm{H}, \mathrm{OCH} 2 \mathrm{CH} 3), 3.96-4.01(\mathrm{~m}, 1 \mathrm{H}, \mathrm{OCH} 2 \mathrm{CH} 3)$, 3.89 (s, 3H, OCH3), 3.71-3.76 (m, 1H, OCH2CH3), 2.24 $(\mathrm{s}, 3 \mathrm{H}, \mathrm{CH} 3), 1.31(\mathrm{t}, 3 \mathrm{H}, J=7.3 \mathrm{~Hz}, \mathrm{CH} 3), 1.29(\mathrm{t}, 3 \mathrm{H}$, $J=7.3 \mathrm{~Hz}, \mathrm{CH} 3) ; 13 \mathrm{C}$ NMR $(125 \mathrm{MHz}, \mathrm{CDCl} 3): 147.45$ (Ar-C), 146.17 (Ar-C), 145.09 (Ar-C), 132.82 (Ar-C), 131.38 (Ar-C), 127.24 (Ar-C), 121.65 (Ar-C), 121.13 (Ar-C), 118.21 (Ar-C), 115.12 (Ar-C), 111.79 (Ar-C), $110.63(\mathrm{Ar}-\mathrm{H}), 63.91$ $\left(\mathrm{d}, J_{\mathrm{PC}}=7.0 \mathrm{~Hz}, \mathrm{OCH} 2 \mathrm{CH} 3\right), 63.73\left(\mathrm{~d}, J_{\mathrm{PC}}=7.0 \mathrm{~Hz} \mathrm{CHP}\right)$, $56.41(\mathrm{OCH} 3), 56.13$ (d, $J=151 \mathrm{~Hz}, \mathrm{CHP}), 17.47(\mathrm{CH} 3)$, $16.85\left(\mathrm{~d}, J_{\mathrm{PCH}}=5.8 \mathrm{~Hz}, \mathrm{CH} 3\right), 16.68\left(\mathrm{~d}, J_{\mathrm{PCH}}=5.75 \mathrm{~Hz}\right.$, CH3); MS: (m/z\%), 413 (M+, 5.2), 276 (100), 137 (10.6).

Diethyl [(5-Chloro-2-methylphenyl amino) (3,4-dimethoxy phenyl) methyl]phosphonate (17). This compound was synthesized after $90 \mathrm{~min}\left(70 \% . \mathrm{Mp}=135.2^{\circ} \mathrm{C}\right) .{ }^{1} \mathrm{H}$ NMR $\left(500 \mathrm{MHz}, \mathrm{CDCl}_{3}\right): 7.02(\mathrm{~s}, 1 \mathrm{H}, \operatorname{ArH}), 6.97(\mathrm{~d}, 2 \mathrm{H}, J=$ $8.1 \mathrm{~Hz}, \operatorname{ArH}), 6.87(\mathrm{~d}, 1 \mathrm{H}, J=8.5 \mathrm{~Hz}, \mathrm{ArH}), 6.64(\mathrm{~d}, 1 \mathrm{H}$, $J=8.1 \mathrm{~Hz}, \mathrm{ArH}), 6.44(\mathrm{~s}, 1 \mathrm{H}, \mathrm{ArH}), 4.69(\mathrm{~d}, 1 \mathrm{H}, J=13.1 \mathrm{~Hz}$, CHP), $4.68(\mathrm{~s}, 1 \mathrm{H}, \mathrm{NH}), 4.1-4.16\left(\mathrm{~m}, 2 \mathrm{H}, \mathrm{OCH}_{2} \mathrm{CH}_{3}\right), 3.96-$ $4.01\left(\mathrm{~m}, 1 \mathrm{H}, \mathrm{OCH}_{2} \mathrm{CH}_{3}\right), 3.9\left(\mathrm{~s}, 6 \mathrm{H}, \mathrm{OCH}_{3}\right), 3.71-3.75(\mathrm{~m}$, $\left.1 \mathrm{H}, \mathrm{OCH}_{2} \mathrm{CH}_{3}\right), 2.24\left(\mathrm{~s}, 3 \mathrm{H}, \mathrm{CH}_{3}\right), 1.32(\mathrm{t}, 3 \mathrm{H}, J=7.3 \mathrm{~Hz}$, $\left.\mathrm{CH}_{3}\right), 1.17\left(\mathrm{t}, 3 \mathrm{H}, J=7.3 \mathrm{~Hz}, \mathrm{CH}_{3}\right) ;{ }^{13} \mathrm{C}$ NMR $(125 \mathrm{MHz}$, $\mathrm{CDCl}_{3}$ ): 149.61 (Ar-C), 149.32 (Ar-C), 145.95 (Ar-C), 132.84 (Ar-C), 131.37 (Ar-C), 128.03 (Ar-C), 121.56 (Ar-C), 12.43 (Ar-C), 118.20 (Ar-C), 111.79 (Ar-C), 111.66 (Ar-C), 111.12 $(\mathrm{Ar}-\mathrm{H}), 63.81\left(\mathrm{~d}, J_{\mathrm{PC}}=6.8 \mathrm{~Hz}, \mathrm{OCH}_{2} \mathrm{CH}_{3}\right), 63.62\left(\mathrm{~d}, J_{\mathrm{PC}}=\right.$ $\left.7.0 \mathrm{~Hz}, \mathrm{OCH}_{2} \mathrm{CH}_{3}\right), 56.38\left(\mathrm{OCH}_{3}\right), 56.27\left(\mathrm{OCH}_{3}\right), 56.11(\mathrm{~d}$, $J=151 \mathrm{~Hz}, \mathrm{CHP}), 17.49(\mathrm{CH} 3), 16.87\left(\mathrm{CH}_{3}\right), 16.72(\mathrm{~d}$, $\left.J_{\mathrm{PCH}}=5.8 \mathrm{~Hz}, \mathrm{CH}_{3}\right), 16.68\left(\mathrm{~d}, J_{\mathrm{PCH}}=5.75 \mathrm{~Hz}, \mathrm{CH}_{3}\right) ; \mathrm{MS}:$ (m/z\%), $427\left(\mathrm{M}^{+}, 6.6\right), 290$ (100), 151 (9.6).

Diethyl [(4-Chloro-2-nitrophenyl amino) (3,4-dimethoxyphenyl) methyl]phosphonate (18). This compound was synthesized after $120 \mathrm{~min}\left(65 \% \mathrm{Mp}=161^{\circ} \mathrm{C}\right) .{ }^{1} \mathrm{H}$ NMR $\left(500 \mathrm{MHz}, \mathrm{CDCl}_{3}\right): 8.87(\mathrm{~d}, 1 \mathrm{H}, J=10.5 \mathrm{~Hz}, \mathrm{ArH}), 8.18$ (s, $1 \mathrm{H}, \mathrm{ArH}), 6.99(\mathrm{~s}, 2 \mathrm{H}, \mathrm{ArH}), 6.87(\mathrm{~d}, 1 \mathrm{H}, J=9.1 \mathrm{~Hz}$, $\operatorname{ArH}), 6.69(\mathrm{~d}, 1 \mathrm{H}, J=9.1 \mathrm{~Hz}, \operatorname{ArH}), 4.8\left(\mathrm{~d}, 1 \mathrm{H}, J_{\mathrm{CHPO}}=\right.$ $23.1 \mathrm{~Hz}, \mathrm{CHP}), 4.09-4.12\left(\mathrm{~m}, 2 \mathrm{H}, \mathrm{OCH}_{2} \mathrm{CH}_{3}\right), 3.97-4.09$ $\left(\mathrm{m}, 2 \mathrm{H}, \mathrm{OCH}_{2} \mathrm{CH}_{3}\right), 4.03-4.06\left(\mathrm{~m}, 2 \mathrm{H}, \mathrm{OCH}_{2} \mathrm{CH}_{3}\right), 3.89$ $\left(\mathrm{s}, 6 \mathrm{H}, \mathrm{OCH}_{3}\right), 1.23-1.32\left(\mathrm{~m}, 6 \mathrm{H}, \mathrm{CH}_{3}\right) ;{ }^{13} \mathrm{C}$ NMR (125 MHz, $\mathrm{CDCl}_{3}$ ): 149.81 (Ar-C), 149.65 (Ar-C), 143.15 (Ar-C), 136.56 (Ar-C), 133.52 (Ar-C), 126.39 (Ar-C), 121.98 (Ar-C), 12030 (Ar-C), 116.66 (Ar-C), 111.72 (Ar-C), 110.88
$(\mathrm{Ar}-\mathrm{C}), 64.16\left(\mathrm{~d}, J_{\mathrm{PC}}=7.2 \mathrm{~Hz}, \mathrm{OCH}_{2} \mathrm{CH}_{3}\right), 63.97\left(\mathrm{~d}, J_{\mathrm{PC}}=\right.$ $\left.6.7 \mathrm{~Hz}, \mathrm{OCH}_{2} \mathrm{CH}_{3}\right), 56.44\left(\mathrm{OCH}_{3}\right), 56.31\left(\mathrm{OCH}_{3}\right), 55.77(\mathrm{~d}$, $\left.J_{\mathrm{PC}}=152 \mathrm{~Hz} C H P\right), 16.84\left(\mathrm{~d}, J_{\mathrm{PCH}}=5.5 \mathrm{~Hz}, \mathrm{CH}_{3}\right), 16.76(\mathrm{~d}$, $\left.J_{\mathrm{PCH}}=5.6 \mathrm{~Hz}, \mathrm{CH}_{3}\right) ; \mathrm{MS}:(\mathrm{m} / \mathrm{z} \%), 458\left(\mathrm{M}^{+}, 1.4\right), 321(100)$, 165 (14).

Diethyl [(4-Methoxy phenyl) (4-nitrophenyl amino) methyl]phosphonate (19). This compound was synthesized after $120 \min \left(76 \% . \mathrm{Mp}=115^{\circ} \mathrm{C}\right) .{ }^{1} \mathrm{H}$ NMR $\left(500 \mathrm{MHz}, \mathrm{CDCl}_{3}\right)$ : $8.0(\mathrm{~d}, 2 \mathrm{H}, J=9.5 \mathrm{~Hz}, \mathrm{ArH}), 7.42(\mathrm{~d}, 2 \mathrm{H}, J=8.7 \mathrm{~Hz}, \mathrm{ArH})$, $6.89(\mathrm{~d}, 2 \mathrm{H}, J=8.5 \mathrm{~Hz}, \mathrm{ArH}), 6.64(\mathrm{~d}, 2 \mathrm{H}, J=9.5 \mathrm{~Hz}$, ArH), $6.37(\mathrm{t}, 1 \mathrm{H}, J=8.5 \mathrm{~Hz}, \operatorname{ArH}), 4.81\left(\mathrm{~d}, 1 \mathrm{H}, J_{\mathrm{CHPO}}=\right.$ $23.5 \mathrm{~Hz}, \mathrm{CHP}), 4.13-4.2\left(\mathrm{~m}, 2 \mathrm{H}, \mathrm{OCH}_{2} \mathrm{CH}_{3}\right), 3.99-3.94(\mathrm{~m}$, $\left.1 \mathrm{H}, \mathrm{OCH}_{2} \mathrm{CH}_{3}\right), 3.79\left(\mathrm{~s}, 3 \mathrm{H}, \mathrm{OCH}_{3}\right), 3.67-3.72(\mathrm{~m}, 1 \mathrm{H}$, $\left.\mathrm{OCH}_{2} \mathrm{CH}_{3}\right), 1.30\left(\mathrm{t}, 3 \mathrm{H}, J=7.3 \mathrm{~Hz}, \mathrm{CH}_{3}\right), 1.16(\mathrm{t}, 3 \mathrm{H}, J=$ $\left.6.6 \mathrm{~Hz}, \mathrm{CH}_{3}\right) ;{ }^{13} \mathrm{C} \mathrm{NMR}\left(125 \mathrm{MHz}, \mathrm{CDCl}_{3}\right): 160.08$ (Ar-C), 152.68 (Ar-C), 139.13 (Ar-C), 129.43 (Ar-C), 126.83 (Ar-C), 126.42 (Ar-C), 114.70 (Ar-C), 112.79 (Ar-C), 64.20 (d, $J_{\mathrm{PC}}=$ $\left.7.0 \mathrm{~Hz}, \mathrm{OCH}_{2} \mathrm{CH}_{3}\right), 63.68\left(\mathrm{~d}, J_{\mathrm{PC}}=7.0 \mathrm{~Hz}, \mathrm{OCH}_{2} \mathrm{CH}_{3}\right)$, $55.67\left(\mathrm{OCH}_{3}\right), 55.29\left(\mathrm{~d},{ }^{3} J_{\mathrm{PC}}=7.5 \mathrm{~Hz}, \underline{\mathrm{CH}}_{3}\right) ; \mathrm{MS}:(\mathrm{m} / \mathrm{z} \%)$, $394\left(\mathrm{M}^{+}, 4\right), 257$ (100), $211(18), 121(3.8)$.

Diethyl [(4-Nitrophenyl)(phenyl amino)methyl]phosphonate (20). This compound was synthesized after 90 min $(73 \%$. $\left.\mathrm{Mp}=77^{\circ} \mathrm{C}\right) .{ }^{1} \mathrm{H}$ NMR $\left(500 \mathrm{MHz}, \mathrm{CDCl}_{3}\right): 8.24(\mathrm{~d}, 2 \mathrm{H}$, $J=9.2 \mathrm{~Hz}, \operatorname{ArH}), 7.70(\mathrm{~d}, 2 \mathrm{H}, J=8.5 \mathrm{~Hz}, \operatorname{ArH}), 7.16(\mathrm{t}$, $1 \mathrm{H}, J=8.2 \mathrm{~Hz}, \mathrm{ArH}), 6.78(\mathrm{t}, 1 \mathrm{H}, J=7.5 \mathrm{~Hz}, \mathrm{ArH}), 6.58(\mathrm{~d}$, $2 \mathrm{H}, J=8.5 \mathrm{~Hz}, \mathrm{ArH}), 4.89\left(\mathrm{~d}, 1 \mathrm{H}, J_{\mathrm{CHPO}}=30.0 \mathrm{~Hz}, \mathrm{CHP}\right)$, 4.87 (s. $1 \mathrm{H}, \mathrm{NH}), 4.17-4.23\left(\mathrm{~m}, 2 \mathrm{H}, \mathrm{OCH}_{2} \mathrm{CH}_{3}\right), 4.06-4.16$ $\left(\mathrm{m}, 1 \mathrm{H}, \mathrm{OCH}_{2} \mathrm{CH}_{3}\right), 3.90-3.95\left(\mathrm{~m}, 1 \mathrm{H}, \mathrm{OCH}_{2} \mathrm{CH}_{3}\right), 1.34$ (t, $\left.3 \mathrm{H}, J=7.3 \mathrm{~Hz}, \mathrm{CH}_{3}\right), 1.23$ (t, $\left.3 \mathrm{H}, J=7.3 \mathrm{~Hz}, \mathrm{CH}_{3}\right)$; ${ }^{13} \mathrm{C}$ NMR (125 MHz, $\mathrm{CDCl}_{3}$ ): 148.07 (Ar-C), 146.06 (Ar-C), 144.49 (Ar-C), 129.79 (Ar-C), 129.06 (Ar-C), 124.19 (ArC), $119.55(\mathrm{Ar}-\mathrm{C}), 114.24(\mathrm{Ar}-\mathrm{C}), 64.16\left(\mathrm{~d}, J_{\mathrm{PC}}=4.8 \mathrm{~Hz}\right.$, $\left.\mathrm{OCH}_{2} \mathrm{CH}_{3}\right), 63.9\left(\mathrm{~d}, J_{\mathrm{PC}}=4.8 \mathrm{~Hz}, \mathrm{OCH}_{2} \mathrm{CH}_{3}\right), 56.48(\mathrm{~d}$, $\left.J_{\mathrm{PC}}=103 \mathrm{~Hz}, \mathrm{CHP}\right), 16.84\left(\mathrm{~d}, J_{\mathrm{PCH} 3}=5.7 \mathrm{~Hz}, \mathrm{CH}_{3}\right), 16.67$ $\left(\mathrm{d}, J_{\mathrm{PCH} 3}=5.5 \mathrm{~Hz}, \mathrm{CH}_{3}\right) ; \mathrm{MS}:(\mathrm{m} / \mathrm{z} \%), 364\left(\mathrm{M}^{+}, 7.9\right), 227$ (100), 181 (23.8), 104 (3.6).

Tetraethyl-1,4-phenylene bis((2-(1H-indolyl)ethylamino)methylene)diphosphonate (21). This compound was previously synthesized [29].

3.2. Modeling. The ligands were drawn in the Hyperchem 8 . The geometry was optimized through the molecular dynamic method AMBER and semiempirical method PM3. The microtubule complexed with paclitaxol was obtained from Protein Data Bank (1JFF).

The Autodock software version 4.2 was used for the molecular docking process. The grids were constructed around the proteins. The Lamarckian Genetic Algorithm method was used for the global optimum binding position search. A number of 100 cycles of calculation were used in order to get a final binding position as accurate as possible. All the compounds as well as griseofulvin were docked into the active site of 1JFF. The complex of ligand-receptor was viewed by Accelry's Discovery Studio Visualizer. The docking 
procedure was run, and the maximum negative $\Delta \mathrm{G}$ was calculated (Table 3 ).

3.3. Antifungal Assay. Microorganisms were obtained from the Mycology and Parasitology Department of the Shiraz University of Medical Sciences. Sabouraud dextrose agar (SDA), potato dextrose agar (PDA), and RPMI 1640 were used for agar dilution and microdilution methods. The clinical isolates of fungi including M. canis, T. mentagrophytes, T. rubrum, E. floccosum, and C. albicans were purified and subcultured on SC, SCC, and PDA media before testing. The stock solution of compounds was prepared in DMSO at a concentration of $200 \mathrm{mg} / \mathrm{mL}$. The compounds were diluted in solid and broth media to obtain final concentration from 0.0625 to $2048 \mu \mathrm{g} / \mathrm{mL}$, using PDA and RPMI 1640 media. The inocula of the molds and yeast were prepared from 210 day mature colonies grown. Fluconazole and griseofulvin were used as positive and the solvents of the compounds as negative blanks.

\section{Conclusion}

$\alpha$-Aminophosphonates are valuable compounds to be investigated as bioactive molecules and pharmacological agents. Recently, we have reported one-pot three-component synthesis starting from aldehydes, amines, and diethylphosphite using $\mathrm{FeCl}_{3}$ as a catalyst to formation of $\alpha$-aminophosphonates [29]. In this study, synthesis of $\alpha$-aminophosphonates using $\mathrm{FeCl}_{3}$ was compared with $\mathrm{CuCl}_{2}$. The results showed that $\mathrm{FeCl}_{3}$ is more efficient than $\mathrm{CuCl}_{2}$ as a catalyst for synthesis of $\alpha$-aminophosphonates.

The biological assays show that only an indole containing bis- $\alpha$-aminophosphonates has antifungal activity against $\mathrm{M}$. canis. The docking results show that these compounds are candidate for cytotoxic activity studies.

\section{Acknowledgments}

This work was supported by Shiraz University of Medical Sciences. The authors are also thankful to Dr. Mohammad Reza Jafari.

\section{References}

[1] R. Engel, "Phosphonates as analogues of natural phosphates," Chemical Reviews, vol. 77, no. 3, pp. 349-367, 1977.

[2] J. Hiratake and J. Oda, "Aminophosphonic and aminoboronic acids as key elements of a transition state analogue inhibitor of enzymes," Bioscience, Biotechnology and Biochemistry, vol. 61, no. 2, pp. 211-218, 1997.

[3] K. Moonen, I. Laureyn, and C. V. Stevens, "Synthetic methods for azaheterocyclic phosphonates and their biological activity," Chemical Reviews, vol. 104, no. 12, pp. 6177-6215, 2004.

[4] F. Palacios, C. Alonso, and J. M. de los Santos, “ $\beta$-phosphonoand phosphinopeptides derived from $\beta$-amino-phosphonic and phosphinic acids," Current Organic Chemistry, vol. 8, no. 15, pp. 1481-1496, 2004.

[5] K. A. Schug and W. Lindner, "Noncovalent binding between guanidinium and anionic groups: focus on biological- and synthetic-based arginine/guanidinium interactions with phosph[on] ate and sulf[on] ate residues," Chemical Reviews, vol. 105, no. 1, pp. 67-113, 2005.

[6] E. K. Baylis, C. D. Campbell, and J. G. Dingwall, "1-Aminoalkylphosphonous acids. Part 1. Isosteres of the protein amino acids," Journal of the Chemical Society, Perkin Transactions 1, pp. 2845-2853, 1984.

[7] D. Ouimette and M. Coffey, "Comparative antifungal activity of four phosphonate compounds against isolates of nine Phytophthora species," Phytopathology, vol. 79, no. 7, pp. 761767, 1989.

[8] S. Yang, X. W. Gao, C. L. Diao et al., "Synthesis and antifungal activity of novel chiral $\alpha$-aminophosphonates containing fluorine moiety," Chinese Journal of Chemistry, vol. 24, no. 11, pp. 1581-1588, 2006.

[9] B. S. Kumar, A. U. R. Sankar, C. Suresh Reddy, S. K. Nayak, and C. Naga Raju, "Synthesis, and antimicrobial activity of 2,10-dichloro-6- substituted aminobenzyl-12H-dibenzo [d, g] [1,3,2] dioxaphosphocin- 6-oxides," Arkivoc, vol. 2007, no. 13, pp. 155-166, 2007.

[10] S. S. Sonar, S. A. Sadaphal, V. B. Labade, B. B. Shingate, and M. S. Shingare, "An efficient synthesis and antibacterial screening of novel oxazepine $\alpha$-aminophosphonates by ultrasound approach," Phosphorus, Sulfur and Silicon and the Related Elements, vol. 185, no. 1, pp. 65-73, 2010.

[11] M. J. Bloemink, J. J. H. Diederen, J. P. Dorenbos, R. J. Heetebrij, B. K. Keppler, and J. Reedijk, "Calcium ions do accelerate the DNA binding of new antitumor-active platinum aminophosphonate complexes," European Journal of Inorganic Chemistry, no. 10, pp. 1655-1657, 1999.

[12] L. Jin, B. Song, G. Zhang et al., "Synthesis, X-ray crystallographic analysis, and antitumor activity of $\mathrm{N}$ (benzothiazole-2-yl)-1-(fluorophenyl)-O,O-dialkyl- $\alpha$-aminophosphonates," Bioorganic and Medicinal Chemistry Letters, vol. 16, no. 6, pp. 1537-1543, 2006.

[13] X. Rao, Z. Song, and L. He, "Synthesis and antitumor activity of novel $\alpha$-aminophosphonates from diterpenic dehydroabietylaminer," Heteroatom Chemistry, vol. 19, no. 5, pp. 512-516, 2008.

[14] Y. Xu, K. Yan, B. Song et al., "Synthesis and antiviral bioactivities of $\alpha$-aminophosphonates containing alkoxyethyl moieties," Molecules, vol. 11, no. 9, pp. 666-676, 2006.

[15] M. R. Saidi and N. Azizi, "A new protocol for a one-pot synthesis of $\alpha$-amino phosphonates by reaction of imines prepared in situ with trialkylphosphites," Synlett, no. 8, pp. 1347-1349, 2002.

[16] B. C. Ranu, A. Hajra, and U. Jana, "General procedure for the synthesis of $\alpha$-amino phosphonates from aldehydes and ketones using indium(III) chloride as a catalyst," Organic Letters, vol. 1, no. 8, pp. 1141-1143, 1999.

[17] A. Manjula, B. V. Rao, and P. Neelakantan, "One-pot synthesis of $\alpha$-aminophosphonates: an inexpensive approach," Synthetic Communications, vol. 33, no. 17, pp. 2963-2969, 2003.

[18] C. Qian and T. Huang, "One-pot synthesis of $\alpha$-amino phosphonates from aldehydes using lanthanide triflate as a catalyst," Journal of Organic Chemistry, vol. 63, no. 12, pp. 4125-4128, 1998.

[19] S. Kumar, S. C. Taneja, M. S. Hundal, and K. K. Kapoor, "Onepot synthesis of $\alpha$-aminophosphonates catalyzed by antimony trichloride adsorbed on alumina," Tetrahedron Letters, vol. 49, no. 14, pp. 2208-2212, 2008.

[20] S. Chandrasekhar, S. J. Prakash, V. Jagadeshwar, and C. Narsihmulu, "Three component coupling catalyzed by $\mathrm{TaCl}_{5}-\mathrm{SiO}_{2}$ : 
synthesis of $\alpha$-amino phosphonates," Tetrahedron Letters, vol. 42, no. 32, pp. 5561-5563, 2001.

[21] T. Akiyama, M. Sanada, and K. Fuchibe, "Bronsted acidmediated synthesis of $\alpha$-amino phosphonates under solventfree conditions," Synlett, no. 10, pp. 1463-1464, 2003.

[22] K. Manabe and S. Kobayashi, "Facile synthesis of $\alpha$-amino phosphonates in water using a Lewis acid- surfactantcombined catalyst," Chemical Communications, no. 8, pp. 669$670,2000$.

[23] H. J. Ha and G. S. Nam, "An efficient synthesis of anilinobenzylphosphonates," Synthetic Communications, vol. 22, no. 8, pp. 1143-1148, 1992.

[24] H. Firouzabadi, N. Iranpoor, and S. Sobhani, "Metal triflatecatalyzed one-pot synthesis of $\alpha$-aminophosphonates from carbonyl compounds in the absence of solvent," Synthesis, no. 16, pp. 2692-2696, 2004.

[25] S. Bhagat and A. K. Chakraborti, "An extremely efficient three-component reaction of aldehydes/ketones, amines, and phosphites (Kabachnik-Fields reaction) for the synthesis of $\alpha$-aminophosphonates catalyzed by magnesium perchlorate," Journal of Organic Chemistry, vol. 72, no. 4, pp. 1263-1270, 2007.

[26] H. Firouzabadi and M. Jafarpour, "Some applications of zirconium(IV) tetrachloride ( $\mathrm{ZrCl} 4$ ) and zirconium(IV) oxydichloride octahydrate $\left(\mathrm{ZrOCl}_{2} \cdot 8 \mathrm{H}_{2} \mathrm{O}\right)$ as catalysts or reagents in organic synthesis," Journal of the Iranian Chemical Society, vol. 5, no. 2, pp. 159-183, 2008.

[27] S. Bhagat and A. K. Chakraborti, "Zirconium(IV) compounds as efficient catalysts for synthesis of $\alpha$-aminophosphonates," Journal of Organic Chemistry, vol. 73, no. 15, pp. 6029-6032, 2008.

[28] M. Hosseini-Sarvari, " $\mathrm{TiO}_{2}$ as a new and reusable catalyst for one-pot three-component syntheses of $\alpha$-aminophosphonates in solvent-free conditions," Tetrahedron, vol. 64, no. 23, pp. 5459-5466, 2008.

[29] Z. Rezaei, H. Firouzabadi, N. Iranpoor et al., "Design and one-pot synthesis of $\alpha$-aminophosphonates and $\operatorname{bis}(\alpha$-aminophosphonates) by iron(III) chloride and cytotoxic activity," European Journal of Medicinal Chemistry, vol. 44, no. 11, pp. 4266-4275, 2009.

[30] K. Rathinasamy, B. Jindal, J. Asthana, P. Singh, P. V. Balaji, and D. Panda, "Griseofulvin stabilizes microtubule dynamics, activates p53 and inhibits the proliferation of MCF-7 cells synergistically with vinblastine," BMC Cancer, vol. 10, pp. 213-226, 2010.

[31] M. Syamala, "Recent progress in three-component reactions. An update," Organic Preparations and Procedures International, vol. 41, no. 1, pp. 1-68, 2009. 


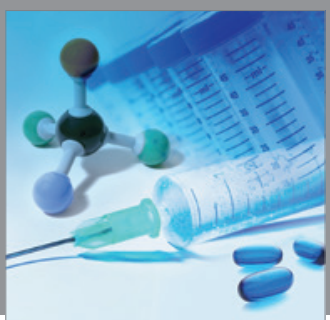

International Journal of

Medicinal Chemistry

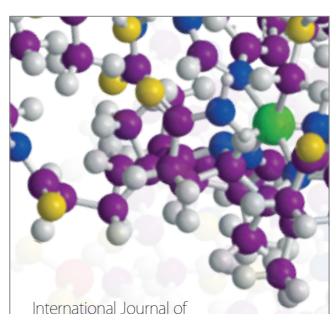

Carbohydrate Chemistry

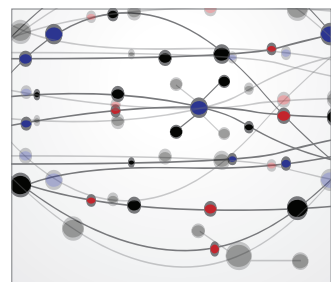

The Scientific World Journal
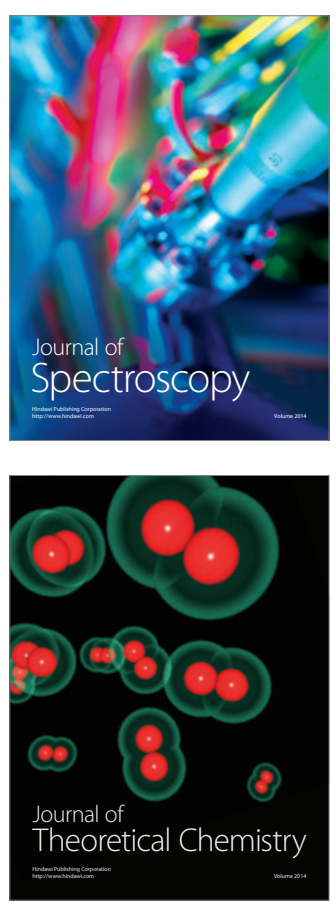
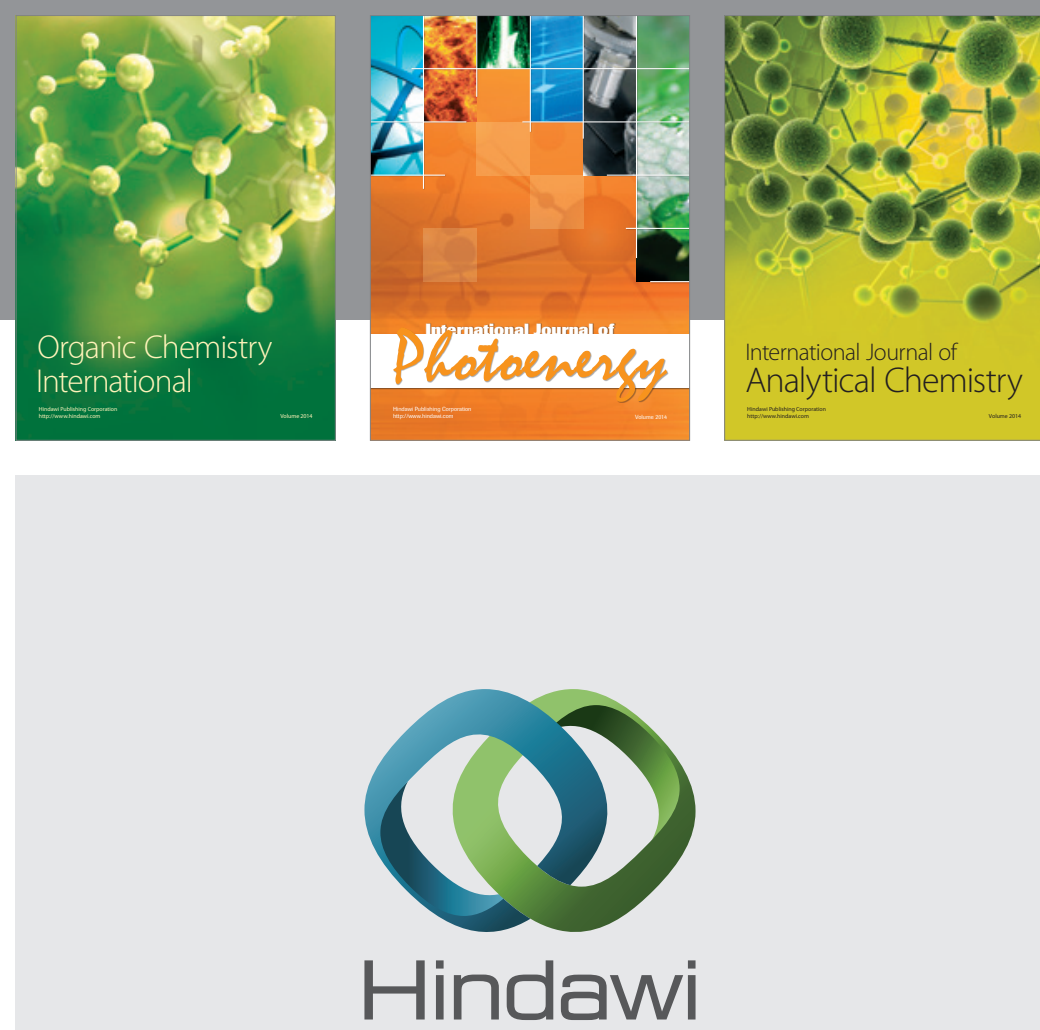

Submit your manuscripts at

http://www.hindawi.com
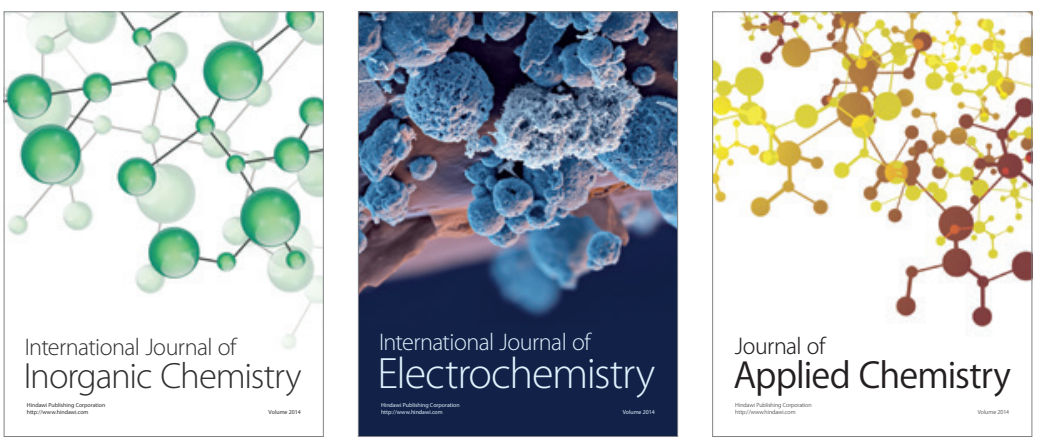

Journal of

Applied Chemistry
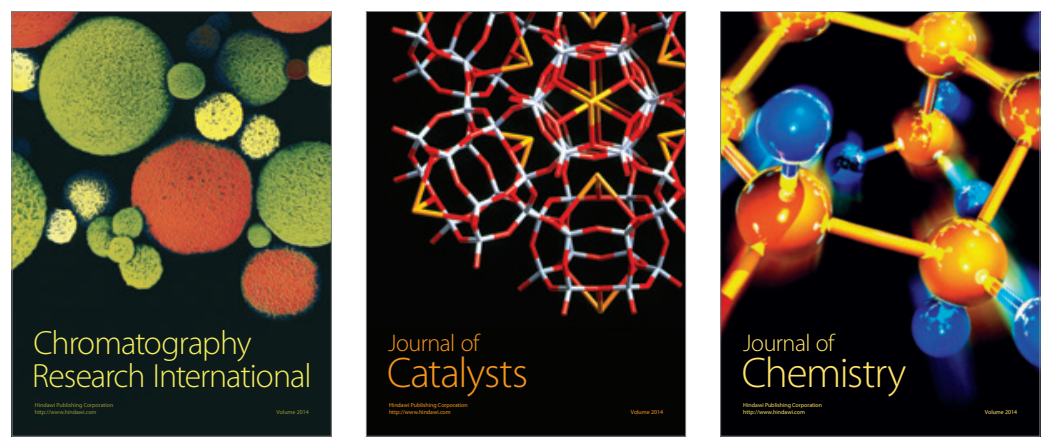
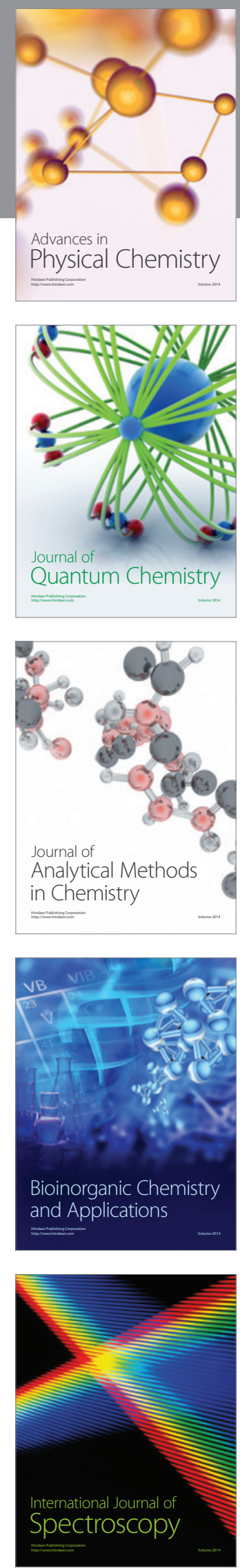